\title{
A Novel Spherical Fuzzy Rough Aggregation Operators Hybrid with TOPSIS Method and Their Application in Decision Making
}

\author{
Chun-Nen Huang $\mathbb{D}^{1},{ }^{1}$ Shahzaib Ashraf $\mathbb{D}^{\mathbb{C}},{ }^{2}$ Noor Rehman $\mathbb{D}^{2},{ }^{2}$ Saleem Abdullah $\mathbb{D}^{3}{ }^{3}$ \\ and Azmat Hussain ${ }^{4}$ \\ ${ }^{1}$ Department of Fire Science, Central Police University, Taoyuan, Taiwan \\ ${ }^{2}$ Department of Mathematics and Statistics, Bacha Khan University, Charsadda, Pakistan \\ ${ }^{3}$ Department of Mathematics, Abdul Wali Khan University, Mardan, Pakistan \\ ${ }^{4}$ Islamic Girls Public School and College, Parachinar, Pakistan \\ Correspondence should be addressed to Noor Rehman; noorrehman@bkuc.edu.pk
}

Received 30 August 2021; Revised 13 October 2021; Accepted 21 October 2021; Published 10 January 2022

Academic Editor: Ewa Rak

Copyright (c) 2022 Chun-Nen Huang et al. This is an open access article distributed under the Creative Commons Attribution License, which permits unrestricted use, distribution, and reproduction in any medium, provided the original work is properly cited.

\begin{abstract}
Industrial control system (ICS) attacks are usually targeted attacks that use the ICS entry approach to get a foothold within a system and move laterally throughout the organization. In recent decades, powerful attacks such as Stuxnet, Duqu, Flame, and Havex have served as wake-up calls for industrial units. All organizations are faced with the rise of security challenges in technological innovations. This paper aims to develop aggregation operators that can be used to address the decision-making problems based on a spherical fuzzy rough environment. Meanwhile, some interesting properties of idempotence, boundedness, and monotonicity for the proposed operators are analyzed. Moreover, we use this newly constructed framework to select ICS security suppliers and validate its acceptability. Furthermore, a different test has been performed based on a new operator to strengthen the suggested approach. Additionally, comparative analysis based on the novel extended TOPSIS method is presented to demonstrate the superiority of the proposed technique. The results show that the conventional approach has a larger area for information representation, better adaptability to the evaluation environment, and higher reliability of the evaluation results.
\end{abstract}

\section{Introduction}

Many governments are launching initiatives to encourage the implementation of electronic and manufacturing innovations, including Germany's industry 4.0 systems, the United States' reindustrialization, and China's "Made in China 2025" strategy to advance next-generation information technology. All of these have has motivated the continuous development of industrial control systems (ICS). All of these have prompted the development of industrial control systems (ICSs) to proceed. Information technology, while adding new development strength to ICS, it also introduces new security flaws. ICS plays a crucial role in the national economy and people's livelihood of crucial national infrastructure. Furthermore, in recent years, a number of notable ICS attacks, such as Stuxnet, Duqu, Flame, and Havex, have raised the concern for authorities and industry sectors. As a result, many manufacturers are working to keep an increasingly open ICS safe. And evaluating ICS security suppliers and selecting the best one are a vital part of management decisions. A typical multi attribute decisionmaking (MADM) problem is selecting an ICS security supplier. MAGDM theories and approaches have stimulated the interest of many researchers in the field of operational research and decision sciences, and significant achievements have been highlighted [1-8]. Scientists have developed several techniques to address the MADM problem, such as the TODIM (an acronym in Portuguese for Interative Multi- 
criteria Decision Making) method [9], MABAC (multi-attributive border approximation area comparison) method [10], EDAS (evaluation based on distance from average solution) method [11], VIKOR (VIekriterijumsko KOmpromisno Rangiranje) method [12], GRA (gray relative analysis) method [13], CODAS (combinative distance-based assessment) method [14], GLDS method [15], QUALIFLEX (qualitative flexible multiple criteria) method [16], and so on. However, as technology advances, experts have observed that the decision-making environment has become more complicated, increasing ambiguity and uncertainty. Trying to solve MADM problems solely with standard tools would fall short of the practical needs [17]. Therefore, several researchers address MADM challenges by combining fuzzy numbers with classical MADM approaches [18]. In this study by Zadeh [19] the fuzzy set syntax was introduced. The membership degree explains "obviously yes" and "certainly no" in its feature. Afterward, the concept of FS has been continually explored and expanded. Atanassov [20] introduced intuitionistic fuzzy sets (IFS) and a novel extension of IFSs known as Pythagorean fuzzy sets (PFSs) [21]. This is well known that the limitation of IFSs is the sum of membership and non-membership that must be less than or equal to one, but PFSs fulfill the criteria that the square sum of membership and non-membership must not be greater than one. PFS provides a broader range of information than IFSs, and PFS-based MAGDM approaches have become a novel and active study field [22-25]. Yager [26] introduced the concept q-rung orthopair fuzzy (q-ROF) set as an extension to the conventional IFS set. The limitation of the q-ROF set is that the sum of the qth-power of membership and non-membership is less than or equal to 1 . Cong and Kreinovich [27] established picture FSs and described operations and relations on them. PFSs deal with three functions: membership, neutral, and non-membership. Manemaran et al. [28] developed a temporal picture fuzzy soft set (PFSS) and addressed its related concepts. Khan et al. [29] presented generalized PFSSs and their application in DM systems. Further, Ashraf et al. [30] introduced the idea of cubic PFSs. Abdullah et al. [31] implemented the cubic PFS approach to address the MADM problem and show it with a numerical example of a petroleum circulation center evaluation problem to demonstrate the usage and application of the proposed ranking technique. It should observe that both IFSs and PFS still have some limitations, although they have been efficient when dealing with complex fuzzy information in some practical applications. The novel idea of spherical fuzzy sets (SFSs) was introduced by Ashraf et al. [8], an advanced tool of FSs, IFSs, and PFSs. They investigated the fundamental characteristics of SFSs and compared them with those of PFSs. Graphically, the spaces of spherical and picture membership grades are investigated. They implemented the aforementioned concept as a practical application to demonstrate the problem of evaluating a food circulation center. Kutlu [32] extended the TOPSIS (Technique for Order of Preference by Similarity to Ideal Solution) method to spherical fuzzy TOPSIS and presented an illustrative example in the MADM problem. Kahraman [33] described q-spherical fuzzy sets and discussed their application in the MADM problem. Zeng [34] discussed T-spherical fuzzy Einstein interactive aggregation operators and their application to selecting photovoltaic cells. Emergency decision making based on SFSs to address the uncertainty in COVID-19 situation is discussed in $[6,7]$.

The rough set (RS) theory, proposed by Professor Pawlak in 1982, is an essential mathematical tool for dealing with ambiguous, inconsistent, and incomplete data and information [35]. The fuzzy rough set (FRS) can be combined with RSs to manage information with continuous attributes and investigate data inconsistencies. The FRS model has shown to be highly effective in many application areas because it is a powerful tool for analyzing inconsistent and ambiguous information. FRS set theory is a rough set theory extension that handles continuous numerical attributes [36]. The significance of the FRS theory may be observed in a variety of applications. Pan et al. [37] established the additive consistent fuzzy preference relation to improve the rough set model of the fuzzy preference relation. Li et al. [38] suggested a practical FRS approach for robust feature selection. Feng et al. [39] reduced multi granulation using uncertainty measures based on FRSs, eliminating the negative and positive regions. Sun et al. [40] used a constructive technique to provide three multi granulation FRSs over two universes. In this study by Liu [41], in the framework of interval-valued fuzzy and fuzzy probabilistic approximation space models, a decision-theoretic RS model was investigated. Zhang used axiomatic and constructive techniques to integrate rough set theory and interval-valued fuzzy set theory and [42] presented a novel paradigm based on FRSs with extended interval values. Zhang et al. [43] offered FR based feature selection based on information entropy to minimize heterogeneous data. By combining granular variable precision FRSs and general fuzzy relations, Wang and $\mathrm{Hu}$ [44] provided a random set of fuzzy relationships. Vluymans et al. [45] introduced a new type of classifier for unbalanced multi-instance data based on FRS theory. Shaheen et al. [46] described the application of generalized hesitant fuzzy rough sets (GHFRS) in risk analysis. Khan et al. [47] addressed the use of a probabilistic hesitant FRS in a decision support system. Tang et al. [48] proposed the decision-theoretic rough set model with q-rung orthopair fuzzy information, as well as its application in evaluating stock investments. Liang et al. [49] suggested q-Rung orthopair fuzzy sets on decisiontheoretic rough sets for three-way decisions under group DM. Zhang et al. [50] proposed group DM using incomplete q-rung orthopair fuzzy preference relations. For MADM, Hussain et al. [51] presented a covering-based q-rung orthopair fuzzy rough set model hybrid with the TOPSIS approach. In practice, these extensions of the q-rung orthopair fuzzy rough set successfully handle DMs' evaluation values in MAGDM problems. Some decision-making techniques are discussed in [53-58].

In this research, motivated by the above discussion, we plan to introduce a new concept of spherical fuzzy rough set (SFRSs). Also, the hybrid technique of aggregation operators and expanded TOPSIS procedure under spherical fuzzy rough setting is presented, in order to benefit of the advantages of the TOPSIS method and SFSs. As a consequence, 
the SFS is the generalized structure of fuzzy set like IF set and PyF set. Thus SFRS is capable of handling more uncertainty than FS, IF set, PyF set, and rough set. Therefore, in this paper, a novel improved TOPSIS-based method and novel algebraic norm-based aggregation operators are established to address with such circumstances of unknown weight information of both DMs and criteria weights and to solve the MAGDM problem after computing all the weights. In order to solve the decision-making problems (DMPs), choosing the ideal opinion, which is better connected to each DMs matrix, is quite essential. In the presented procedure, ideal opinion is nominated under the SFR average method. Generalized distance measure is established to find the differences between two SFRSs. In the presented SFR TOPSIS and SFR aggregation operators for solving MAGDM problems, generalized distance measures-based entropy measure is introduced to find out the criteria weights under SFR information used in this paper. The challenges in the industrial control systems security and sustainability are addressed by using proposed advance methodology.

In summary, the main contributions of the present study are follows:

(1) Novel idea of spherical fuzzy rough aggregation operators namely spherical fuzzy rough weighted averaging, spherical fuzzy rough ordered weighted averaging and spherical fuzzy rough hybrid weighted averaging operators is introduced and their basic operational laws are investigated.

(2) A case study of industrial control security service provider selection is also presented to demonstrate the applicability of the established operators.

(3) To validate the findings, different test on aggregation operators are implemented.

(4) Finally, comparisons with the spherical fuzzy rough TOPSIS method are made to interpret the outcomes. The ranking of the obtained results is presented graphically.

\section{Preliminaries}

In this section, we will put forward some basic literature concerning IFS, PFS, SFS, relation and rough sets, which will be helpful for subsequent sections.

Definition 1 (see [20]). Consider a universal set $\mathcal{S}$. An IFS $\mathscr{G}$ on a set $\mathcal{S}$ is defined as

$$
\mathscr{G}=\left\{s, \xi_{\mathscr{G}}(s), \psi_{\mathscr{G}}(s) \mid s \in \mathcal{S}\right\},
$$

where $\xi_{\mathscr{G}}: \mathcal{S} \longrightarrow[0,1]$ and $\psi_{\mathscr{G}}: \mathcal{S} \longrightarrow[0,1]$ denotes the MemD and NMemD of an object $s \in \mathcal{S}$ to the set $\mathscr{G}$ such that $0 \leq \xi_{\mathscr{G}}(s)+\psi_{\mathscr{G}}(s) \leq 1$. For an alternative $s \in \mathcal{S}, \pi_{\mathscr{G}}(s)=1-$ $\left(\xi_{\mathscr{G}}(s)+\psi_{\mathscr{G}}(s)\right)$ is known as the degree of hesitancy.

Definition 2 (see [27]). Assume a universal set $\mathcal{S}$. A PFS $\mathscr{G}$ on a set $\mathcal{S}$ is of the form as follows:

$$
\mathscr{G}=\left\{\left\langle s, \xi_{\mathscr{G}}(s), \eta_{\mathscr{G}}(s), \psi_{\mathscr{G}}(s)\right\rangle \mid s \in \mathcal{S}\right\},
$$

where

$$
\xi_{\mathscr{g}}: \mathcal{S} \longrightarrow[0,1], \eta_{\mathscr{g}}: \mathcal{S} \longrightarrow[0,1]
$$

and $\psi_{\mathscr{g}}: \mathcal{S} \longrightarrow[0,1]$ denotes the positive membership (PM), neutral membership (NeuM) and negative membership (NM) of an object $s \in \mathcal{S}$ to the set $\mathscr{G}$ such that $0 \leq \xi_{\mathscr{G}}(s)+$ $\eta_{\mathscr{G}}(s)+\psi_{\mathscr{G}}(s) \leq 1$. For an alternative $s \in \mathcal{S}$, $\pi_{\mathscr{G}}(s)=1-\left(\xi_{\mathscr{G}}(s)+\eta_{\mathscr{G}}(s)+\psi_{\mathscr{G}}(s)\right)$ is known as the degree of hesitancy.

Definition 3 (see [8]). Assume a universal set $\mathcal{S}$. A SFS $\mathscr{G}$ on a set $\mathcal{S}$ is of the form as follows:

$$
\mathscr{G}=\left\{\left\langle s, \xi_{\mathscr{G}}(s), \eta_{\mathscr{G}}(s), \psi_{\mathscr{G}}(s)\right\rangle \mid s \in \mathcal{S}\right\},
$$

where

$$
\xi_{\mathscr{\varphi}}: \mathcal{S} \longrightarrow[0,1], \eta_{\mathscr{\varphi}}: \mathcal{S} \longrightarrow[0,1]
$$

$\psi_{\mathscr{g}}: \mathcal{S} \longrightarrow[0,1]$ denote the PM, NeuM and NM of an object $s \in \mathcal{S}$ to the set $\mathscr{G}$ such that $0 \leq\left(\xi_{\mathscr{G}}(s)\right)^{2}+\left(\eta_{\mathscr{G}}(s)\right)^{2}+$ $\left(\psi_{\mathscr{G}}(s)\right)^{2} \leq 1$. For an alternative $s \in \mathcal{S}$, the degree of hesitancy is given as $\pi_{\mathscr{G}}(s)=$ $\sqrt{1-\left(\left(\xi_{\mathscr{G}}(s)\right)^{2}+\left(\eta_{\mathscr{G}}(s)\right)^{2}+\left(\psi_{\mathscr{G}}(s)\right)^{2}\right)}$.

Consider $\quad \mathscr{G}=\left\langle s, \xi_{\mathscr{G}}(s), \eta_{\mathscr{G}}(s), \psi_{\mathscr{G}}(s)\right\rangle \quad$ denotes $\mathscr{G}=\left(\xi_{\mathscr{G}}, \eta_{\mathscr{G}}, \psi_{\mathscr{G}}\right)$ known as a SP values (SPV) if there is no confusion. The family of subsets of SFS is represented by $(\mathrm{SFS}(\mathcal{S}))$.

Definition 4 (see [8]). Let $\mathscr{G}_{1}=\left\langle\xi_{\mathscr{G}_{1}}, \eta_{\mathscr{G}_{1}}, \psi_{\mathscr{G}_{1}}\right\rangle$, and $\mathscr{G}_{2}=$ $\left\langle\xi_{\mathscr{G}_{2}}, \eta_{\mathscr{G}_{2}}, \psi_{\mathscr{G}_{2}}\right\rangle$ be any two SFVs. Then the basic operations are given as, for $\lambda>1$.

(i) $\mathscr{G}_{1} \bigcup \mathscr{G}_{2}=\left\{\max \left\langle\xi_{\mathscr{G}_{1}}(s), \xi_{\mathscr{G}_{2}}(s)\right\rangle, \min \left\langle\eta_{\mathscr{G}_{1}}(s), \eta\right.\right.$ $\left.\left.\mathscr{G}_{2}(s)\right\rangle, \min \left\langle\psi_{\mathscr{S}_{1}}(s), \psi_{\mathscr{S}_{2}}(s)\right\rangle \mid s \in \mathcal{S}\right\}$.

(ii) $\mathscr{G}_{1} \cap \mathscr{G}_{2}=\left\{\min \left\langle\xi_{\mathscr{G}_{1}}(s), \xi_{\mathscr{G}_{2}}(s)\right\rangle, \min \langle\eta\right.$ $\left.\left.\mathscr{G}_{1}(s), \eta_{\mathscr{G}_{2}}(s)\right\rangle, \max \left\langle\psi_{\mathscr{G}_{1}}(s), \psi_{\mathscr{G}_{2}}(s)\right\rangle \mid s \in \mathcal{S}\right\}$,

(iii) $\mathscr{G}_{1} \subseteq \mathscr{G}_{2}$ if for all $s \in \mathcal{S}, \xi_{\mathscr{G}_{1}} \leq \xi_{\mathscr{G}_{2}}, \eta_{\mathscr{G}_{1}} \leq \eta_{\mathscr{G}_{2}}$, and $\psi_{\mathscr{G}_{1}} \geq \psi_{\mathscr{G}_{2}}$,

(iv) $\mathscr{G}_{1} \oplus \mathscr{G}_{2}=\left(\sqrt{\xi_{\mathscr{G}_{1}}^{2}+\xi_{\mathscr{G}_{2}}^{2}-\xi_{\mathscr{G}_{1}}^{2} \xi_{\mathscr{G}_{2}}^{2}}, \eta_{\mathscr{G}_{1}} \eta_{\mathscr{G}_{2}}, \psi_{\mathscr{G}_{1}} \psi_{\mathscr{G}_{2}}\right)$,

(v) $\mathscr{G}_{1} \otimes \mathscr{G}_{2}=\left(\xi_{\mathscr{G}_{1}} \xi_{\mathscr{G}_{2}}, \eta_{\mathscr{G}_{1}} \eta_{\mathscr{G}_{2}}\right.$, $\sqrt{\left.\psi_{\mathscr{G}_{1}}^{2}+\psi_{\mathscr{G}_{2}}^{2}-\psi_{\mathscr{G}_{1}}^{2} \psi_{\mathscr{G}_{2}}^{2}\right)}$

(vi) $\lambda \mathscr{G}_{1}=\left(\sqrt{1-\left(1-\xi_{\mathscr{G}_{1}}^{2}\right)^{\lambda}}, \eta_{\mathscr{G}_{1}}^{\lambda}, \psi_{\mathscr{G}_{1}}^{\lambda}\right)$,

(vii) $\mathscr{G}_{1}^{\lambda}=\left(\xi_{\mathscr{G}_{1}}^{\lambda}, \eta_{\mathscr{G}_{1}}^{\lambda}, \sqrt{1-\left(1-\psi_{\mathscr{G}_{1}}^{2}\right)^{\lambda}}\right)$.

Definition 5. Suppose a universal set $\mathcal{S}$ and let $g \in \mathcal{S} \times \mathcal{S}$ be any binary relation. Then

(i) $g$ is reflexive if $(s, s) \in g, \forall s \in \mathcal{S}$;

(ii) $g$ is symmetric if $\forall s, r \in \mathcal{S},(s, r) \in g$ then $(r, s) \in g$;

(iii) $g$ is transitive if $\forall s, r, t \in \mathcal{S},(s, r) \in g$ and $(r, t) \in g$, then $(s, t) \in g$.

Definition 6. Let $\mathcal{S}$ be a universal set and $g \in \mathcal{S} \times \mathcal{S}$ be an arbitrary binary relation on set $\mathcal{S}$. Then $g^{*}$ denotes the set value mapping given as $g^{*}: \mathcal{S} \longrightarrow \mathscr{P}(\mathcal{S})$, where

$$
g^{*}(s)=\{r \in \mathcal{S} \mid(s, r) \in g\}, \quad \text { for } s \in \mathcal{S} .
$$


The set $g^{*}(s)$ is said to be a successor neighborhood of an element $s$ with respect to $g$. The pair $(\mathcal{S}, g)$ represents the approximation space with respect to $g^{*}$ Consider an approximation space $(\mathcal{S}, g)$ and $b$ be any subset of $\mathcal{S}$, then lower and upper approximation of $b$ is represented and defined as follows:

$$
\begin{aligned}
& g(b)=\left\{s \in \mathcal{S} \mid g^{*}(s) \subseteq b\right\}, \\
& \dot{g}(b)=\left\{s \in \mathcal{S} \mid g^{*}(s) \cap b \neq \varnothing\right\} .
\end{aligned}
$$

Therefore, the pair $(g(b), ' g(b))$ is called rough set. Then the mapping ' $g(b), g(b): \mathscr{P}(\mathcal{S}) \longrightarrow \mathscr{P}(\mathcal{S})$ denotes the approximation operators.

\section{Spherical Fuzzy Rough Sets}

SFS is the most significant generalization of IFS, PyFS and PFS which provide more space for experts for assigning values to PM, NeuM and NM. Here we will present the hybrid notion of SFS and rough sets to get the novel concept of SF rough set. The new score and accuracy mapping are defined for the developed model and studied their desirable properties of the presented model with detail.

Definition 7. Suppose a universal set $\mathcal{S}$ and let $\mathbf{g} \in S \mathscr{F} S(\mathcal{S} \times$ $\mathcal{S})$ be SF relation. Then

(i) $g$ is reflexive if $\xi_{g}(s, s)=1, \eta_{g}(s, s)=0$, and $\psi_{g}(s, s)=0, \forall s \in \mathcal{S}$

(ii) $g$ is symmetric if $\forall(s, r) \in \mathcal{S} \times \mathcal{S}$, $\xi_{g}(r, s)=\xi_{g}(s, r), \quad \eta_{g}(r, s)=\eta_{g}(s, r), \quad$ and $\psi_{g}(r, s)=\psi_{g}(s, r)$;

(iii) $g$ is transitive if $\forall(s, t) \in \mathcal{S} \times \mathcal{S}$, $\xi_{g}(r, t) \geq \underset{s \in \mathcal{S}}{\vee}\left[\xi_{g}(r, s) \wedge \xi_{g}(s, t)\right], \quad \eta_{g}(r, t)=\wedge_{s \in \mathcal{S}}$ $\left[\eta_{g}(r, s) \vee \eta_{g}(r, s)\right], \quad$ and $\quad \psi_{g}(r, t)=\wedge_{s \in \mathcal{S}}\left[\psi_{g}(r, s) \vee\right.$ $\left.\psi_{g}(s, t)\right]$.

Alternatively, the relation $g$, is transitive if it holds the following:

For all $s, r \in \mathcal{S}$ and $\lambda_{1}, \lambda_{2}, \lambda_{3} \in[0,1]$

(a) $\forall t \in \mathcal{S}, \xi_{g}(r, s) \geq \lambda_{1}$, $\xi_{g}(s, t) \geq \lambda_{1} \Longrightarrow \xi_{g}(r, t) \geq \lambda_{1}$,

and

(b) $\forall t \in \mathcal{S}, \eta_{g}(r, s) \leq \lambda_{2}$, and $\eta_{g}(s, t) \leq \lambda_{2} \Longrightarrow \eta_{g}(r, t) \leq$ $\lambda_{2}$, (c) $\forall t \in \mathcal{S}, \psi_{g}(r, s) \leq \lambda_{3}$, and $\psi_{g}(s, t) \leq \lambda_{3} \Longrightarrow \psi_{g}(r, t) \leq$
$\lambda_{3}$.

Definition 8. Suppose a universal set $\mathcal{S}$ and let $g \in S \mathscr{F} S(\mathcal{S} \times \mathcal{S})$ be any SF relation. The pair $(\mathcal{S}, g)$ denotes a SF approximation space. Let $b$ be any subset of $S \mathscr{F} S(\mathcal{S})$ i.e. $b \subseteq I \mathscr{F} S(\mathcal{S})$. Then based on SF approximation space $(\mathcal{S}, g)$, the upper and lower approximations of $b$ are denoted by ' $g(b)$ and $g(b)$ and are given as follows:

$$
\begin{aligned}
& \dot{g}^{\prime}(b)=\left\{\left\langle s, \xi_{g^{\prime}(b)}(s), \eta_{g^{\prime}(b)}(s), \psi_{g^{\prime}(b)}(s)\right\rangle \mid s \in \mathcal{S}\right\} \\
& g(b)=\left\{\left\langle s, \xi_{g_{(b)}}(s), \eta_{g_{(b)}}(s), \psi_{g_{(b)}}(s)\right\rangle \mid s \in \mathcal{S}\right\},
\end{aligned}
$$

where

$$
\begin{aligned}
\xi_{g^{\prime}(b)}(s) & =\vee_{t \in \mathcal{S}}\left[\xi_{g}(s, t) \vee \xi_{g}(t)\right], \\
\eta_{g^{\prime}(b)}(s) & =\wedge_{t \in \mathcal{S}}\left[\eta_{g}(s, t) \wedge \eta_{g}(t)\right], \\
\psi_{g^{\prime}(b)}(s) & =\wedge_{t \in \mathcal{S}}\left[\psi_{g}(s, t) \wedge \psi_{g}(t)\right], \\
\xi_{g(b)}(s) & =\wedge_{t \in \mathcal{S}}\left[\xi_{g}(s, t) \wedge \xi_{g}(t)\right], \\
\eta_{g(b)}(s) & =\wedge_{t \in \mathcal{S}}^{\wedge}\left[\eta_{g}(s, t) \wedge \eta_{g}(t)\right], \\
\psi_{g(b)}(s) & =\vee_{t \in \mathcal{S}}\left[\psi_{g}(s, t) \vee \psi_{g}(t)\right] .
\end{aligned}
$$

such that $0 \leq\left(\xi_{\bar{g}(b)}(s)\right)^{2}+\left(\eta_{\bar{g}(b)}(s)\right)^{2}+\left(\psi_{\bar{g}(b)}(s)\right)^{2} \leq 1$ and $0 \leq\left(\xi_{g(b)}(s)\right)^{2}+\left(\eta_{g(b)}(s)\right)^{2}+\left(\psi_{g(b)}(s)\right)^{2} \leq 1$. As $\quad g(b)$ and $g(b)$ are SFSs, so ' $g(b), g(b): S \mathscr{F} S(\mathcal{S}) \longrightarrow S \mathscr{F} S(\mathcal{S})$ are upper and lower approximation operators. Therefore, the pair $\quad g(b)=\left(g(b),{ }^{\prime} g(b)\right)=\left\{\left\langle s,\left(\xi_{g(b)}(s), \eta_{g(b)}(s)\right.\right.\right.$, $\left.\left.\left.\psi_{g_{(b)}}(s)\right),\left(\xi_{g^{\prime}(b)}(s), \eta_{g^{\prime}(b)}(s), \psi_{g^{\prime}(b)}\right)\right\rangle \mid s \in b\right\}$ is called SF rough set. For simplicity $g(b)=(g(b), ' g(b))=$ $\left\{\left\langle s,\left(\xi_{g_{(}(b)}(s), \psi_{g_{(b)}}(s)\right),\left(\xi_{g^{\prime}(b)}(s), \psi_{g^{\prime}(b)}\right)\right\rangle \mid s \in b\right\}$ is denoted as $g(b)=(\underline{g}(b), \bar{g}(b))=((\underline{\xi}, \underline{\eta}, \underline{\psi}),(\bar{\xi}, \bar{\eta}, \bar{\psi}))$ known as SF rough number (SFRN).

Example 1. Consider a fixed set $\mathcal{S}=\left\{s_{1}, s_{2}, s_{3}, s_{4}, s_{5}\right\}$. Let $(\mathcal{S}, g)$ be an SF approximation space and let $g \in S \mathscr{F} S(\mathcal{S} \times$ $\mathcal{S}$ ) be a SF relation which is given in Table 1 ,

Consider the professional experts presented the optimum decision normal object in the form of SFS, that is,

$$
b=\left\{\left\langle s_{1}, 0.8,0.1,0.4\right\rangle,\left\langle s_{2}, 0.6,0.3,0.5\right\rangle,\left\langle s_{3}, 0.9,0.2,0.3\right\rangle,\left\langle s_{4}, 0.7,0.3,0.4\right\rangle,\left\langle s_{5}, 0.4,0.3,0.5\right\rangle\right\} .
$$

Now to calculate $\underline{g}(b)$ and $\underline{g}(b)$, we have 
TABle 1: SF relation from set from $S \times S$.

\begin{tabular}{lcccrr}
\hline$g$ & $S_{1}$ & $S_{2}$ & $S_{3}$ & $S_{4}$ & $S_{5}$ \\
\hline$S_{1}$ & $(0.6,0.3,0.1)$ & $(0.7,0.6,0.2)$ & $(0.6,0.5,0.5)$ & $(0.7,0.3,0.6)$ & $(0.6,0.3,0.1)$ \\
$S_{2}$ & $(0.7,0.6,0.2)$ & $(0.9,0.1,0.3)$ & $(0.8,0.4,0.3)$ & $(0.5,0.2,0.4)$ & $(0.5,0.6,0.3)$ \\
$S_{3}$ & $(0.8,0.3,0.4)$ & $(0.3,0.5,0.6)$ & $(0.4,0.7,0.5)$ & $(0.6,0.7,0.3)$ & $(0.7,0.6,0.2)$ \\
$S_{4}$ & $(0.5,0.2,0.6)$ & $(0.6,0.4,0.2)$ & $(0.8,0.4,0.3)$ & $(0.9,0.2,0.3)$ & $(0.8,0.2,0.4)$ \\
$S_{1}$ & $(0.9,0.2,0.3)$ & $(0.7,0.2,0.6)$ & $(0.2,0.5,0.3)$ & $(0.4,0.1,0.3)$ & $(0.6,0.5,0.4)$ \\
\hline
\end{tabular}

$$
\begin{aligned}
\xi_{\bar{g}(b)}\left(s_{1}\right)= & \underset{t \in \mathcal{S}}{\vee}\left[\xi_{g}(s, t) \vee \xi_{b}(t)\right], \\
= & (0.6 \vee 0.8) \vee(0.7 \vee 0.6) \vee(0.6 \vee 0.9) \\
& \vee(0.7 \vee 0.7) \vee(0.6 \vee 0.4)=0.9 . \\
\eta_{\bar{g}(b)}\left(s_{1}\right)= & \wedge_{t \in \mathcal{S}}\left[\eta_{g}(s, t) \wedge \eta_{b}(t)\right] \\
= & (0.3 \wedge 0.1) \wedge(0.6 \wedge 0.3) \wedge(0.5 \wedge 0.2) \\
& \wedge(0.3 \wedge 0.3) \wedge(0.3 \wedge 0.3)=0.1 . \\
\psi_{\bar{g}(b)}\left(s_{1}\right)= & \wedge \underset{t \in \mathcal{S}}{ }\left[\psi_{g}(s, t) \wedge \psi_{b}(t)\right] \\
= & (0.1 \wedge 0.4) \wedge(0.2 \wedge 0.5) \wedge(0.5 \wedge 0.3) \wedge(0.6 \wedge 0.4) \\
& \wedge(0.1 \wedge 0.5)=0.1 .
\end{aligned}
$$

$\begin{aligned} & \text { So, } \\ & (0.9,0.1,0.1) .\end{aligned}\left(\overline{\xi_{1}}, \overline{\eta_{1}}, \overline{\psi_{1}}\right)=\left(\xi_{\bar{g}(b)}\left(s_{1}\right), \eta_{\bar{g}(b)}\left(s_{1}\right), \psi_{\bar{g}(b)}\left(s_{1}\right)\right)=$

Likewise, we can calculate the others values of upper and lower approximation:

$$
\begin{aligned}
\left(\overline{\xi_{2}}, \overline{\eta_{2}}, \overline{\psi_{2}}\right) & =(0.9,0.1,0.2),\left(\overline{\xi_{3}}, \overline{\eta_{3}}, \overline{\psi_{3}}\right)=(0.9,0.1,0.2),\left(\overline{\xi_{4}}, \overline{\eta_{4}}, \overline{\psi_{4}}\right)=(0.9,0.1,0.2) \\
\left(\overline{\xi_{5}}, \overline{\eta_{5}}, \overline{\psi_{5}}\right) & =(0.9,0.1,0.3) \\
\left(\underline{\xi_{1}}, \underline{\eta_{1}}, \underline{\psi_{1}}\right) & =(0.4,0.1,0.7) \\
\left(\underline{\xi_{2}}, \underline{\eta_{2}}, \underline{\psi_{2}}\right) & =(0.4,0.1,0.5),\left(\underline{\xi_{3}}, \underline{\eta_{3}}, \underline{\psi_{3}}\right)=(0.3,0.1,0.6),\left(\underline{\xi_{4}}, \underline{\eta_{4}}, \underline{\psi_{4}}\right)=(0.4,0.1,0.6),\left(\underline{\xi_{5}}, \underline{\eta_{5}}, \underline{\psi_{5}}\right)=(0.2,0.1,0.6), \\
\bar{g}(b) & =\left\{\left(s_{1}, 0.9,0.1,0.1\right),\left(s_{2}, 0.9,0.1,0.2\right),\left(s_{3}, 0.9,0.1,0.2\right),\left(s_{4}, 09 ., 0.1,0.2\right),\left(s_{5}, 0.9,0.1,0.3\right)\right\} \\
\underline{g}(b) & =\left\{\left(s_{1}, 0.4,0.1,0.7\right),\left(s_{2}, 0.4,0.1,0.5\right),\left(s_{3}, 0.3,0.1,0.6\right),\left(s_{4}, 0.4,0.1,0.6\right),\left(s_{5}, 0.2,0.1,0.6\right)\right\} \\
g(b) & =(\underline{g}(b), \bar{g}(b))=\left\{\begin{array}{r}
\left.s_{1},(0.4,0.1,0.7),(0.9,0.1,0.1), s_{2},(0.4,0.1,0.5),(0.9,0.1,0.2), s_{3},(0.3,0.1,0.6),(0.9,0.1,0.2)\right) \\
s_{4},(0.4,0.1,0.6),\left(s_{4}, 09 ., 0.1,0.2\right), s_{5},(0.2,0.1,0.6),(0.9,0.1,0.3)
\end{array}\right.
\end{aligned}
$$

Definition 9. Assume two SFRNs $g\left(b_{1}\right)=\left(g\left(b_{1}\right), \bar{g}\left(b_{1}\right)\right)$ and $g\left(b_{2}\right)=\left(g\left(b_{2}\right), \bar{g}\left(b_{2}\right)\right)$. Then some basic operations on them are given as follows:

(i) $\underline{g}\left(b_{1}\right) \bigcup g\left(b_{2}\right)=\left\{\left(\underline{g}\left(b_{1}\right) \cup \underline{g}\left(b_{2}\right)\right),\left(\bar{g}\left(b_{2}\right) \bigcup\right.\right.$ $\left.\left.\bar{g}\left(b_{2}\right)\right)\right\}$;

(ii) $g\left(b_{1}\right) \cap g\left(b_{2}\right)=\left\{\left(\underline{g}\left(b_{1}\right) \cap \underline{g}\left(b_{2}\right)\right),\left(\bar{g}\left(b_{1}\right)\right.\right.$ $\left.\left.\cap \bar{g}\left(b_{2}\right)\right)\right\}$

(iii) $g\left(b_{1}\right) \oplus g\left(b_{2}\right)=\left\{\left(\underline{g}\left(b_{1}\right) \oplus \underline{g}\left(b_{2}\right)\right),\left(\bar{g}\left(b_{1}\right) \oplus \bar{g}\left(b_{2}\right)\right)\right\}$;

(iv) $g\left(b_{1}\right) \oplus g\left(b_{2}\right)=\left\{\left(\underline{g}\left(b_{1}\right) \oplus \underline{g}\left(b_{2}\right)\right),\left(\bar{g}\left(b_{1}\right) \oplus \bar{g}\left(b_{2}\right)\right)\right\}$;

(v) $g\left(b_{1}\right) \subseteq g\left(b_{2}\right)=\left(\underline{g}\left(b_{1}\right) \subseteq \underline{g}\left(b_{2}\right)\right)$ and $\left(\bar{g}\left(b_{1}\right)\right.$ $\left.\subseteq \bar{g}\left(b_{2}\right)\right)$;

(vi) $\lambda g\left(b_{1}\right)=\left(\lambda \underline{g}\left(b_{1}\right), \lambda \bar{g}\left(b_{1}\right)\right)$ for $\lambda \geq 1$; (vii) $\left(g\left(b_{1}\right)\right)^{\lambda}=\left(\left(\underline{g}\left(b_{1}\right)\right)^{\lambda},\left(\bar{g}\left(b_{1}\right)\right)^{\lambda}\right)$ for $\lambda \geq 1$;

(viii) $g\left(b_{1}\right)^{c}=\left(\underline{g}\left(b_{1}\right)^{c}, \bar{g}\left(b_{1}\right)^{c}\right)$ where $\quad g\left(b_{1}\right)^{c}$ and $\bar{g}\left(b_{1}\right)^{c}$ denotes the complement of SF rough approximation operators $g\left(b_{1}\right)$ and $\bar{g}\left(b_{1}\right)$, i.e. $\bar{g}\left(b_{1}\right)^{c}=(\psi, \eta, \underline{\xi})$ and $\bar{g}\left(b_{1}\right)^{c}=(\bar{\psi}, \bar{\eta}, \bar{\xi})$

(ix) $g\left(b_{1}\right)=\bar{g}\left(b_{2}\right)$ iff $\underline{g}\left(b_{1}\right)=\underline{g}\left(b_{2}\right)$ and $\bar{g}\left(b_{1}\right)=\bar{g}\left(b_{2}\right)$.

Definition 10. Let the SFRN $g(b)=(g(b), \bar{g}(b))=$ $((\underline{\xi}, \underline{\eta}, \underline{\psi}),(\bar{\xi}, \bar{\eta}, \bar{\psi}))$. Then the score function defined as:

$$
\widetilde{S}(g(b))=\frac{1}{6}(4+\underline{\xi}+\bar{\xi}-\underline{\eta}-\underline{\psi}-\bar{\eta}-\bar{\psi}), \widetilde{S}(g(b)) \in[0,1] .
$$


The accuracy of the SFRN $g(b)=(\underline{g}(b), \bar{g}(b))=$ $((\underline{\xi}, \underline{\eta}, \underline{\psi}),(\bar{\xi}, \bar{\eta}, \bar{\psi}))$ for $g(b)$ is defined as:

$$
\mathbb{A}(g(b))=\frac{1}{2}(\underline{\xi}+\bar{\xi}-\underline{\psi}-\bar{\psi}), \mathscr{A}(g(b)) \in[-1,1] .
$$

Consider two SFRNs $g\left(b_{1}\right)=\left(\underline{g}\left(b_{1}\right), \bar{g}\left(b_{1}\right)\right)$ and $g\left(b_{2}\right)=\left(\underline{g}\left(b_{2}\right), \bar{g}\left(b_{2}\right)\right)$, then

(i) If $\widetilde{S}\left(g\left(b_{1}\right)\right)>\widetilde{S}\left(g\left(b_{2}\right)\right)$, then $g\left(b_{1}\right)>R\left(b_{2}\right)$,

(ii) If $\widetilde{S}\left(g\left(b_{1}\right)\right)<\widetilde{S}\left(g\left(b_{2}\right)\right)$, then $g\left(b_{1}\right)<R\left(b_{2}\right)$,

(iii) If $\widetilde{S}\left(g\left(b_{1}\right)\right)=\widetilde{S}\left(g\left(b_{2}\right)\right)$, then

(a) If $\mathbb{A}\left(g\left(b_{1}\right)\right)>A\left(g\left(b_{2}\right)\right)$, then $g\left(b_{1}\right)>R\left(b_{2}\right)$,

(b) If $\mathbb{A}\left(g\left(b_{1}\right)\right)<A\left(g\left(b_{2}\right)\right)$, then $g\left(b_{1}\right)<R\left(b_{2}\right)$,

(c) If $\mathbb{A}\left(g\left(b_{1}\right)\right)=\mathbb{A}\left(g\left(b_{2}\right)\right)$, then $g\left(b_{1}\right)=g\left(b_{2}\right)$.

Proposition 1. Assume for any two SFRNs $g\left(b_{1}\right)=\left(g\left(b_{1}\right), \bar{g}\left(b_{1}\right)\right)$ and $g\left(b_{2}\right)=\left(g\left(b_{2}\right), \bar{g}\left(b_{2}\right)\right)$ with respect to $\bar{S} F$ approximation space $(\mathcal{S}, g)$. Then the following properties are hold for SFRNs.

(i) $\sim\left(\sim g\left(b_{1}\right)\right)=b_{1}$, where $\sim g\left(b_{1}\right)$ is the complement of $g\left(b_{1}\right)$;

(ii) $g\left(b_{1}\right) \cup g\left(b_{2}\right)=g\left(b_{2}\right) \cup g\left(b_{1}\right)$ and $g\left(b_{1}\right) \cap$ $g\left(b_{2}\right)=g\left(b_{2}\right) \cap g\left(b_{1}\right)$

(iii) $\sim\left(g\left(b_{1}\right) \bigcup g\left(b_{2}\right)\right)=\left(\sim g\left(b_{1}\right)\right) \cap\left(\sim g\left(b_{2}\right)\right)$;

(iv) $\sim\left(g\left(b_{1}\right) \cap g\left(b_{2}\right)\right)=\left(\sim g\left(b_{1}\right)\right) \bigcup\left(\sim g\left(b_{2}\right)\right)$;

(v) If $b_{1} \subseteq b_{2}$, then $g\left(b_{1}\right) \subseteq g\left(b_{2}\right)$;

(vi) $g\left(b_{1} \bigcup b_{2}\right) \supseteq g\left(b_{1}\right) \cup g\left(b_{2}\right)$;

(vii) $g\left(b_{1} \cap b_{2}\right) \subseteq g\left(b_{1}\right) \cap g\left(b_{2}\right)$.

\section{Spherical Fuzzy Rough Averaging Aggregation Operators}

Aggregation operators have the ability to aggregative the collective information of several professional experts into a single value. Here we will put forward the hybrid study of SFRS and averaging aggregation operators to get SFR averaging aggregation operators and presented their desirable properties.

4.1. Spherical Fuzzy Rough Weighted Averaging Aggregation Operators. This subsection is devoted for the study of SPRWA aggregation operators and presented the fundamental properties of SFRWA operators.

Definition 11. Let $g\left(b_{i}\right)=\left(g\left(b_{i}\right), \bar{g}\left(b_{i}\right)\right)(i=1,2, \ldots, n)$ be the collection of SFRNs. Assume $u=\left(u_{1}, u_{2}, \ldots, u_{n}\right)^{T}$ be the weight vectors with $\sum_{i=1}^{n} u_{i}=1$ and $0 \leq u_{i} \leq 1$. Then the SFRWA aggregation operators are given as follows:

$\operatorname{SFRWA}\left(g\left(b_{1}\right), g\left(b_{2}\right), \ldots, g\left(b_{n}\right)\right)=\left(\oplus_{i=1}^{n} u_{i} \underline{g}\left(b_{i}\right), \oplus_{i=1}^{n} u_{i} \bar{g}\left(b_{i}\right)\right)$.

The aggregation result for the above definition is described in Theorem 1.

Theorem 1. Consider $g\left(b_{i}\right)=\left(g\left(b_{i}\right), \bar{g}\left(b_{i}\right)\right)(i=1,2, \ldots$, $n)$ be the collections of SFRNs. Consider the weight vectors $u=\left(u_{1}, u_{2}, \ldots, u_{n}\right)^{T}$ with $\sum_{i=1}^{n} u_{i}=1$ and $0 \leq u_{i} \leq 1$. Then the aggregation result for SFRWA operator is given as follows:

$$
\begin{aligned}
& \operatorname{SFRWA}\left(g\left(b_{1}\right), g\left(b_{1}\right), \ldots, g\left(b_{n}\right)\right)=\left\{\oplus_{i=1}^{n} u_{i} \underline{g}\left(b_{i}\right), \oplus_{i=1}^{n} u_{i} \bar{g}\left(b_{i}\right)\right\}
\end{aligned}
$$

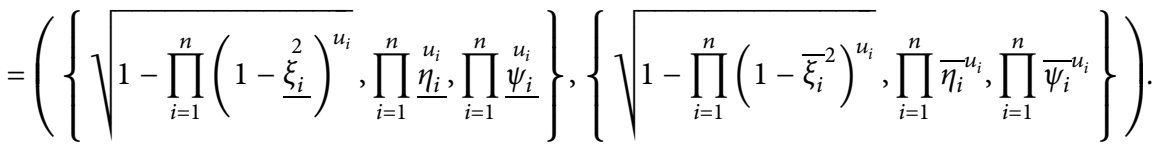

Proof. To get the required proof, we will use mathematical induction.

By using the operation law, we have

$$
\begin{aligned}
& g\left(b_{1}\right) \oplus g\left(b_{2}\right)=\left(\underline{g}\left(b_{1}\right) \oplus \underline{g}\left(b_{2}\right), \bar{g}\left(b_{1}\right) \oplus \bar{g}\left(b_{2}\right)\right)
\end{aligned}
$$

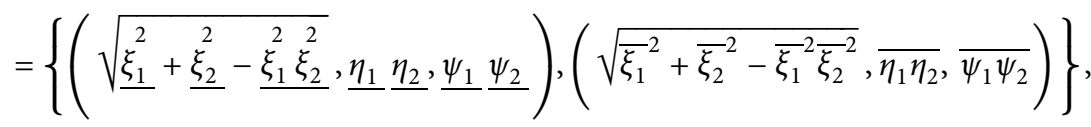

$$
\begin{aligned}
& \lambda \underline{g}\left(b_{1}\right)=\left(\lambda \underline{g}\left(b_{1}\right), \lambda \bar{g}\left(b_{1}\right)\right)
\end{aligned}
$$

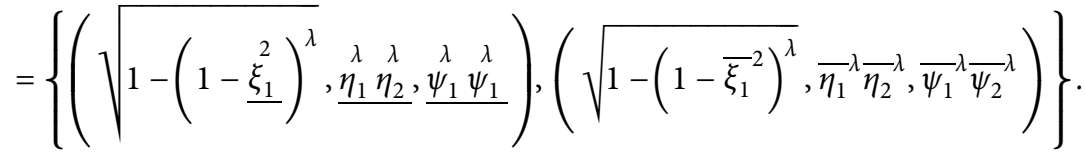


Suppose $n=2$, then

$$
\begin{aligned}
& \operatorname{SFRWA}\left(g\left(b_{1}\right), g\left(b_{2}\right)\right)=\left[\oplus_{i=1}^{2} u_{i} \underline{g}\left(b_{i}\right), \oplus_{i=1}^{2} u_{i} \bar{g}\left(b_{i}\right)\right]
\end{aligned}
$$

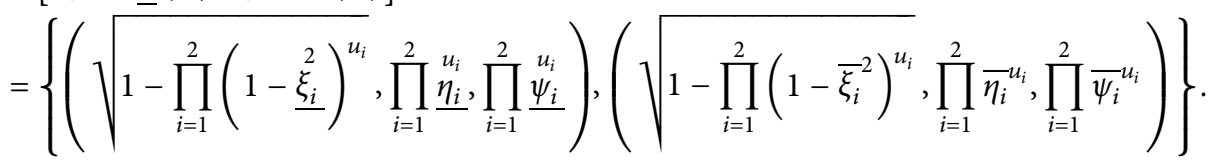

Thus, the result holds for $n=2$.

Now suppose that result is true for $n=k$

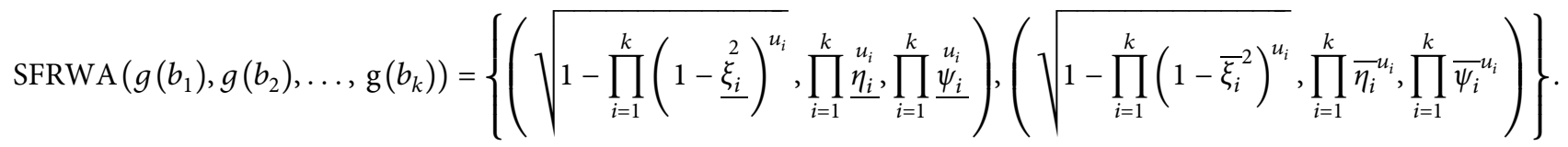

Let us show that the result holds for $n=k+1$, thus we have

$$
\begin{aligned}
& \operatorname{SFRWA}\left\{\left(g\left(b_{1}\right), g\left(b_{2}\right), \ldots, \mathrm{g}\left(b_{k}\right)\right), g\left(b_{k+1}\right)\right\}=\left[\left\{\left(\oplus_{i=1}^{k} u_{i} \underline{g}\left(b_{i}\right)\right) \oplus\left(b_{k+1} \underline{g}\left(b_{k+1}\right)\right)\right\},\left\{\left(\oplus_{i=1}^{k} u_{i} \bar{g}\left(b_{i}\right)\right) \oplus\left(b_{k+1} \bar{g}\left(b_{k+1}\right)\right)\right\}\right]
\end{aligned}
$$

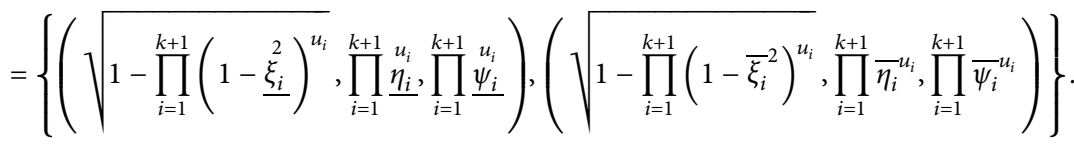

This show that the result is true for $n=k+1$. Therefore, the result is hold for all $n \geq 1$.

Based on Theorem $1, g(b)$ and $\bar{g}(b)$ are SFRNs. Therefore, $\oplus_{i=1}^{n} u_{i} g\left(b_{i}\right)$ and $\oplus_{i=1}^{n} u_{i} \bar{g}\left(b_{i}\right)$ are also SFRNs. Thus, it is clear that SFRWA $\left(g\left(b_{1}\right), \ldots, g\left(b_{n}\right)\right)$ is also a SFRN based on $\mathrm{SF}$ approximation space $(\mathcal{S}, g)$.

Some fundamental and desirable properties of SFRWA operator are presented in Theorem 2.

Theorem 2. Let $g\left(b_{i}\right)=\left(g\left(b_{i}\right), \bar{g}\left(b_{i}\right)\right)(i=1,2, \ldots, n)$ be the collections of SFRNs with $i=\left(i_{1}, i_{2}, \ldots, i_{n}\right)^{T}$ with $\sum_{i=1}^{n} u_{i}=1$ and $0 \leq u_{i} \leq 1$. Then SFRWA operator satisfies the following the properties.

(i) Idempotency: if $g\left(b_{i}\right)=g(\mathscr{L})$ for all $i=1,2, \ldots, n$ where $g(\mathscr{L})=(g(\mathscr{L}), \bar{g}(\mathscr{L}))=((\underline{r}, \underline{s}, \underline{t}), \quad(\bar{r}, \bar{s}$, $\bar{t}))$, then $\operatorname{SFRW} \bar{A}\left(g\left(b_{1}\right), g\left(b_{2}\right), \ldots, g\left(b_{n}\right)\right)=g(\mathscr{L})$. (ii) Monotonicity: consider that $g\left(\mathscr{L}_{i}\right)=\left(g\left(\mathscr{L}_{i}\right)\right.$, $\left.\bar{g}\left(\mathscr{L}_{i}\right)\right)(i=1,2, \ldots, n)$ be another family of $S F R V s$ with $g\left(\mathscr{L}_{i}\right) \leq g\left(b_{i}\right)$ and $\bar{g}\left(\mathscr{L}_{i}\right) \leq \bar{g}\left(b_{i}\right)$, then $\operatorname{SFRW} \bar{A}\left(g\left(\mathscr{L}_{1}\right), g\left(\mathscr{L}_{2}\right)\right.$, $\left.\ldots, g\left(\mathscr{L}_{n}\right)\right) \leq \operatorname{SFRWA}\left(g\left(b_{1}\right), g\left(b_{2}\right), \ldots, g\left(b_{n}\right)\right)$.

(iii) Boundedness: let $(g(b))^{-}=\left(\min _{i} \underline{g}\left(b_{i}\right), \max _{i} \bar{g}\left(b_{i}\right)\right)$ and $(g(b))^{+}=\left(\min _{i} g\left(b_{i}\right), \max _{i} \bar{g}\left(b_{i}\right)\right)$, then $(g(b))^{-} \leq \operatorname{SFRWA}\left(g\left(b_{1}\right), g\left(b_{2}\right), \ldots, \quad g\left(b_{n}\right)\right) \leq$ $\left(g\left(b_{i}\right)\right)^{+}$.

Proof

(i) Idempotency: as $g\left(b_{i}\right)=g(\mathscr{L})$ (for all $\left.=1,2, \ldots, n\right)$, where $g(\mathscr{L})=(\underline{g}(\mathscr{L}), \bar{g}(\mathscr{L}))=((\underline{r}, \underline{s}, \underline{t}),(\bar{r}, \bar{s}$, $\bar{t})$ )

$$
\begin{aligned}
& \operatorname{SFRWA}\left(g\left(b_{1}\right), \ldots, g\left(b_{n}\right)\right)=\left[\oplus_{i=1}^{n} u_{i} \underline{g}\left(b_{i}\right), \oplus_{i=1}^{n} u_{i} \bar{g}\left(b_{i}\right)\right]
\end{aligned}
$$

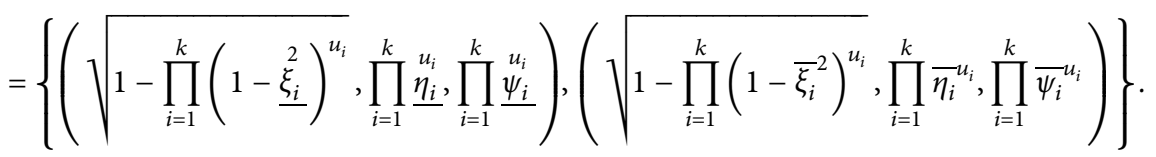


For all $\quad i,\left(b_{i}\right)=g(\mathscr{L})=(\underline{g}(\mathscr{L}), \bar{g}(\mathscr{L}))=$ $((\underline{r}, \underline{s}, \underline{t}),(\bar{r}, \bar{s}, \bar{t}))$. Therefore,

$$
\begin{aligned}
& ((\underline{r}, \underline{s}, \underline{t}),(\bar{r}, \bar{s}, \bar{t})) \underline{g}, \bar{g}
\end{aligned}
$$

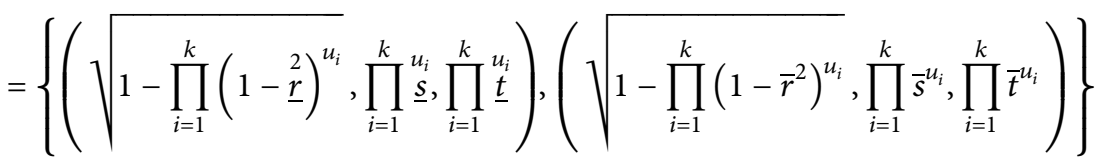

$$
\begin{aligned}
& =\{((\underline{r}, \underline{s}, \underline{t}),(\bar{r}, \bar{s}, \bar{t}))\} \\
& =(\underline{g}(\mathscr{L}), \bar{g}(\mathscr{L}))=g(\mathscr{L}) \text {. }
\end{aligned}
$$

Hence,

$$
\operatorname{SFRWA}\left(g\left(b_{1}\right), \ldots, g\left(b_{n}\right)\right)=g(\mathscr{L}) .
$$

(ii) Monotonicity: since $g\left(\mathscr{L}_{i}\right)=\left(\underline{g}\left(\mathscr{L}_{i}\right), \bar{g}\left(\mathscr{L}_{i}\right)\right)=$ $\left(\left(\underline{r}_{i}, \underline{s}_{i}, \underline{t}_{i}\right),\left(\bar{r}_{i}, \bar{s}_{i}, \bar{t}_{i}\right)\right)$ and $g\left(b_{i}\right)=\left(g\left(b_{i}\right), \bar{g}\left(\mathscr{L}_{i}\right)\right)$ and $\underline{g}\left(\mathscr{L}_{i}\right) \leq \underline{g}\left(b_{i}\right)$ and $\bar{g}\left(\mathscr{L}_{i}\right) \leq \bar{g}\left(\bar{b}_{i}\right)$ (for $i=1$, $2, \ldots, n)$,

$$
\begin{aligned}
& \underline{r_{i}} \leq \underline{\xi_{i}} \Longrightarrow 1-\underline{\xi_{i}} \leq 1-\underline{r_{i}^{2}} \Longrightarrow \prod_{i=1}^{n}\left(1-\underline{\xi_{i}^{2}}\right)^{u_{i}} \leq \prod_{i=1}^{n}\left(1-\underline{r_{i}}\right)^{u_{i}} \\
& \Longrightarrow 1-\prod_{i=1}^{n}\left(1-\underline{r_{i}^{2}}\right)^{u_{i}} \leq 1-\prod_{i=1}^{n}\left(1-\underline{\xi_{i}^{2}}\right)^{u_{i}} \Longrightarrow \sqrt{1-\prod_{i=1}^{n}\left(1-\underline{r_{i}}\right)^{u_{i}}} \leq \sqrt{1-\prod_{i=1}^{n}\left(1-\underline{\xi_{i}}\right)^{u_{i}}} .
\end{aligned}
$$

Further,

$$
\underline{s_{i}} \leq \underline{\eta_{i}} \Longrightarrow \underline{u_{i}} \leq \underline{\eta_{i}} \Longrightarrow \prod_{i=1}^{n} \underline{u_{i}} \leq \prod_{i=1}^{u_{i}} \underline{\eta_{i}} .
$$

Next,

$$
\underline{t_{i}} \geq \underline{\psi_{i}} \Longrightarrow \prod_{i=1}^{n} \underline{t_{i}} \geq \prod_{i=1}^{n} \underline{u_{i}} .
$$

Similarly, we can show that

$$
\begin{aligned}
1-\prod_{i=1}^{n}\left(1-\overline{r_{i}}\right)^{u_{i}} & \leq 1-\prod_{i=1}^{n}\left(1-\overline{\xi_{i}}\right)^{u_{i}}, \\
\prod_{i=1}^{n} \bar{S}_{i}^{u_{i}} & \leq \prod_{i=1}^{n} \bar{\eta}_{i}^{u_{i}}, \\
\prod_{i=1}^{n}{\overline{r_{i j}}}^{u_{i}} & \geq \prod_{i=1}^{n} \bar{\psi}_{i j}^{u_{i}} .
\end{aligned}
$$

Thus, from the above calculation, it is cleared that

$$
\underline{g}\left(\mathscr{L}_{i}\right) \leq \underline{g}\left(b_{i}\right) \text { an } d \bar{g}\left(\mathscr{L}_{i}\right) \leq \bar{g}\left(b_{i}\right) .
$$

Therefore,

$$
\begin{aligned}
& \operatorname{SFRWA}\left(g\left(\mathscr{L}_{1}\right), g\left(\mathscr{L}_{2}\right), \ldots, g\left(\mathscr{L}_{n}\right)\right) \\
& \quad \leq \operatorname{SFRWA}\left(g\left(b_{1}\right), g\left(b_{2}\right), \ldots, g\left(b_{n}\right)\right) .
\end{aligned}
$$

(iii) Boundedness: proof is easy and follow from (i) and (ii).

4.2. Spherical Fuzzy Rough Ordered Weighted Averaging Aggregation Operators. This subsection is devoted for the study of SPROWA aggregation operators, which weigh the ordered position of the argument. Then we have presented the fundamental properties of SFROWA operators.

Definition 12. Let $g\left(b_{i}\right)=\left(g\left(b_{i}\right), \bar{g}\left(b_{i}\right)\right)(i=1,2, \ldots, n)$ be the collection of SFRNs. Assume $u=\left(u_{1}, u_{2}, \ldots, u_{n}\right)^{T}$ be the weight vectors with $\sum_{i=1}^{n} u_{i}=1$ and $0 \leq u_{i} \leq 1$. Then the SFROWA aggregation operators are given as follows: 


$$
\begin{gathered}
\operatorname{SFROWA}\left(g\left(b_{1}\right), g\left(b_{2}\right), \ldots, g\left(b_{n}\right)\right) \\
=\left(\oplus_{i=1}^{n} u_{i} \underline{g_{\delta}}\left(b_{i}\right), \oplus_{i=1}^{n} u_{i} \overline{g_{\delta}}\left(b_{i}\right)\right) .
\end{gathered}
$$

The aggregation result for the above definition is described in Theorem 3.
Theorem 3. Consider $g\left(b_{i}\right)=\left(g\left(b_{i}\right), \bar{g}\left(b_{i}\right)\right)(i=1,2$, $\ldots, n)$ be the collections of SFRNs. Consider the weight vectors $u=\left(u_{1}, u_{2}, \ldots, u_{n}\right)^{T}$ with $\sum_{i=1}^{n} u_{i}=1$ and $0 \leq u_{i} \leq 1$. Then the aggregation result for SFROWA operator given is as follows:

$$
\begin{aligned}
& \operatorname{SFROWA}\left(g\left(b_{1}\right), g\left(b_{1}\right), \ldots, g\left(b_{n}\right)\right)=\left\{\oplus_{i=1}^{n} u_{i} \underline{g_{\delta}}\left(b_{i}\right), \oplus_{i=1}^{n} u_{i} \overline{g_{\delta}}\left(b_{i}\right)\right\}
\end{aligned}
$$

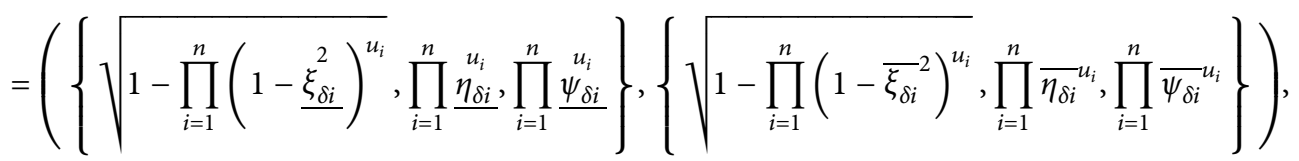

whereg $_{\delta}\left(b_{i}\right)=\left(\underline{g_{\delta}}\left(b_{i}\right), \overline{g_{\delta}}\left(b_{i}\right)\right)$ denotes the largest value of permutation from the collection $g\left(b_{i}\right)$.

Proof. Proof is easy and followed from Theorem 1.

Some fundamental and desirable properties of SFROWA operator are presented in Theorem 4.

Theorem 4. Let $g\left(b_{i}\right)=\left(g\left(b_{i}\right), \bar{g}\left(b_{i}\right)\right)(i=1,2, \ldots, n)$ be the collections of SFRNs with $u=\left(u_{1}, u_{2}, \ldots, u_{n}\right)^{T}$ with $\sum_{i=1}^{n} u_{i}=1$ and $0 \leq u_{i} \leq 1$. Then SFROWA operator satisfies the following the properties.

(i) Idempotency: if $g\left(b_{i}\right)=g(\mathscr{L})$ for all $=1,2, \ldots, n$ where-

$g(\mathscr{L})=(g(\mathscr{L}), \bar{g}(\mathscr{L}))=((\underline{r}, \underline{s}, \underline{t}),(\bar{r}, \bar{s}, \bar{t}))$.

Then SFROWA $\left(g\left(b_{1}\right), g\left(b_{2}\right), \ldots, g\left(b_{n}\right)\right)=g(\mathscr{L})$.

(ii) Monotonicity: consider that $g\left(\mathscr{L}_{i}\right)=$ $\left(g\left(\mathscr{L}_{i}\right), \bar{g}\left(\mathscr{L}_{i}\right)\right)(i=1,2, \ldots, n)$ be another family of $S \bar{F} R V s$ with $\underline{g}\left(\mathscr{L}_{i}\right) \leq \underline{g}\left(b_{i}\right)$ and $\bar{g}\left(\mathscr{L}_{i}\right) \leq \bar{g}\left(b_{i}\right)$, then

$$
\begin{aligned}
& \operatorname{SFROWA}\left(g\left(\mathscr{L}_{1}\right), g\left(\mathscr{L}_{2}\right), \ldots, g\left(\mathscr{L}_{n}\right)\right) \\
& \quad \leq \operatorname{SFROWA}\left(g\left(b_{1}\right), g\left(b_{2}\right), \ldots, g\left(b_{n}\right)\right) .
\end{aligned}
$$

(iii) Boundedness: let $(g(b))^{-}=\left(\min g\left(b_{i}\right), \min \bar{g}\left(b_{i}\right)\right)$ and $(g(b))^{+}=\left(\max g\left(b_{i}\right), \max \bar{g}\left(\bar{b}_{i}\right)\right), \quad i \quad$ then $\left.(g(b))^{-} \leq \operatorname{SFROW}{ }^{i} \overline{(g}\left(b_{1}\right),{ }^{i} \quad g\left(b_{2}\right), \ldots, g\left(b_{n}\right)\right) \leq$ $\left(g\left(b_{i}\right)\right)^{+}$.

Proof. Proofs directly followed from Theorem 2.
4.3. Spherical Fuzzy Rough Hybrid Averaging Aggregation Operators. SFRHA operators are the significant generalization of SFRWA and SFROWA aggregation operators because it has the ability to weight both the ordered position and the argument itself. In this subsection, we will investigate the study of SPRHA aggregation operators and presented the fundamental properties of SFRHA aggregation operators.

Definition 13. Let $g\left(b_{i}\right)=\left(g\left(b_{i}\right), \bar{g}\left(b_{i}\right)\right)(i=1,2, \ldots, n)$ be the collection of SFRNs with weight vector $v=\left(v_{1}, v_{2}, \ldots, v_{n}\right)^{T} \quad$ such that $\sum_{i=1}^{n} v_{i}=1$ and $0 \leq v_{i} \leq 1$. Consider $u=\left(u_{1}, u_{2}, \ldots, u_{n}\right)^{T}$ be the associated weight vectors with $\sum_{i=1}^{n} u_{i}=1$ and $0 \leq u_{i} \leq 1$. Then the SFRHA aggregation operators are given as follows:

$$
\begin{array}{r}
\operatorname{SFRHA}\left(g\left(b_{1}\right), g\left(b_{2}\right), \ldots, g\left(b_{n}\right)\right) \\
=\left(\oplus_{i=1}^{n} u_{i} \underline{\widetilde{g}_{\delta}}\left(b_{i}\right), \oplus_{i=1}^{n} u_{i} \underline{\tilde{g}_{\delta}}\left(b_{i}\right)\right) .
\end{array}
$$

The aggregation result for above definition is described in Theorem 3.

Theorem 5. Consider $g\left(b_{i}\right)=\left(g\left(b_{i}\right), \bar{g}\left(b_{i}\right)\right)(i=1,2, \ldots$, $n)$ be the collections of $S F R \bar{N} s$ with weight vector $v=\left(v_{1}, v_{2}, \ldots, v_{n}\right)^{T}$ such that $\sum_{i=1}^{n} v_{i}=1$ and $0 \leq v_{i} \leq 1$. Consider $u=\left(u_{1}, u_{2}, \ldots, u_{n}\right)^{T}$ be the associated weight vectors with $\sum_{i=1}^{n} u_{i}=1$ and $0 \leq u_{i} \leq 1$. Then the aggregation result for SFRHA operator given as:

$$
\begin{aligned}
& \operatorname{SFRHA}\left(g\left(b_{1}\right), g\left(b_{1}\right), \ldots, g\left(b_{n}\right)\right)=\left\{\oplus_{i=1}^{n} u_{i} \underline{\widetilde{g}_{\delta}}\left(b_{i}\right), \oplus_{i=1}^{n} u_{i} \underline{\widetilde{g}_{\delta}}\left(b_{i}\right)\right\} \\
& \quad=\left(\left\{\sqrt{1-\prod_{i=1}^{n}\left(1-\underline{\tilde{\xi}_{\delta i}^{2}}\right)^{u_{i}}}, \prod_{i=1}^{n} \underline{\tilde{\eta}_{\delta i}}, \prod_{i=1}^{n} \underline{\tilde{\psi}_{\delta i}}\right\},\left\{\sqrt{\left.\left.1-\prod_{i=1}^{n}\left(1-\overline{\widetilde{\xi}}_{\delta i}^{2}\right)^{u_{i}}, \prod_{i=1}^{n} \overline{\widetilde{\eta}}_{\delta i}^{u_{i}}, \prod_{i=1}^{n} \widetilde{\widetilde{\psi}}_{\delta i}^{u_{i}}\right\}\right),}\right.\right.
\end{aligned}
$$


where $\widetilde{g}_{\delta}\left(b_{i}\right)=\left(\widetilde{g}_{\delta}\left(b_{i}\right), \overline{\tilde{g}_{\delta}}\left(b_{i}\right)\right)$ denotes the largest value of permutation from the collection $g\left(b_{i}\right)$.

Proof. Proof is easy and followed from Theorem 1.

Some fundamental and desirable properties of SFRHA operator is presented in Theorem 6.

Theorem 6. Let $g\left(b_{i}\right)=\left(g\left(b_{i}\right), \bar{g}\left(b_{i}\right)\right)(i=1,2, \ldots, n)$ be the collections of SFRNs with weight vector $v=\left(v_{1}, v_{2}, \ldots, v_{n}\right)^{T}$ such that $\sum_{i=1}^{n} v_{i}=1$ and $0 \leq v_{i} \leq 1$. Consider $u=\left(u_{1}, u_{2}, \ldots, u_{n}\right)^{T}$ be the associated weight vectors with $\sum_{i=1}^{n} u_{i}=1$ and $0 \leq u_{i} \leq 1$. Then SFRHA operator satisfies the following the properties.

(i) Idempotency: if $g\left(b_{i}\right)=g(\mathscr{L})$ for all $=1,2, \ldots, n$ where $g(\mathscr{L})=(\underline{g}(\mathscr{L}), \bar{g}(\mathscr{L}))=((\underline{r}, \underline{s}, \underline{t}),(\bar{r}$,

$\bar{s}, \bar{t}))$ then SFRHA $\left(g\left(b_{1}\right), g\left(b_{2}\right), \ldots\right.$, $\left.g\left(b_{n}\right)\right)=g(\mathscr{L})$.

(ii) Monotonicity: consider that $g\left(\mathscr{L}_{i}\right)=\left(g\left(\mathscr{L}_{i}\right), \bar{g}\left(\mathscr{L}_{i}\right)\right)(i=1,2, \ldots, n)$ be another family of SFRVs with $g\left(\mathscr{L}_{i}\right) \leq g\left(b_{i}\right)$ and $\bar{g}\left(\mathscr{L}_{i}\right) \leq \bar{g}\left(b_{i}\right)$, then $\operatorname{SFRHA}\left(g\left(\mathscr{L}_{1}\right), \bar{g}\left(\mathscr{L}_{2}\right), \ldots\right.$, $\left.g\left(\mathscr{L}_{n}\right)\right) \leq \operatorname{SFRHA}\left(g\left(b_{1}\right), g\left(b_{2}\right), \ldots, g\left(b_{n}\right)\right)$. (iii) Boundedness: let $(g(b))^{-}=\left(\min g\left(b_{i}\right), \min \bar{g}\left(b_{i}\right)\right)$ and $(g(b))^{+}=\left(\max g\left(b_{i}\right), \max ^{i} \bar{g}\left(\bar{b}_{i}\right)\right)$, then $(g(b))$ $-\leq \operatorname{SFRHA}\left(g\left(b_{1}\right)^{i}, g\left(b_{2}\right), \ldots,, g\left(b_{n}\right)\right) \leq\left(g\left(b_{i}\right)\right)^{+}$.

Proof. Proofs directly followed from Theorem 2.

\section{Decision Support Algorithm}

Here, we have developed a framework for addressing uncertainty in decision making (DM) under spherical fuzzy rough information. Consider a DM problem with $\left\{\beth_{1}, \beth_{2}, \ldots, \beth_{g}\right\}$ be a set of alternative and $\left\{\neg_{1}, \neg_{2}, \ldots, \neg_{h}\right\}$ be a set of attributes with $\left(\partial_{1}, \partial_{2}, \ldots, \partial_{h}\right)$ be the weight vector, such that each $\partial_{t} \epsilon[0,1], \sum_{t=1}^{n} \partial_{t}=1$. To test the reliability of kth alternative $\beth_{k}$ under the $t$ th attribute $\tau_{t}$, let a set of decision makers (DMs) $\left\{D_{1}, D_{2}, \ldots, D_{j}\right\}$ and $\left(\eta_{1}, \eta_{2}, \ldots, \eta_{j}\right)$ be DMs weights such that $\eta_{t} \in[0,1], \sum_{t=1}^{j} \eta_{t}=1$. The expert evaluation matrix is described as follows:

$$
M=\left[g^{j}(b)\right]_{g \times h}=\left[\begin{array}{cccc}
\left(\underline{g}\left(b_{11}\right), \bar{g}\left(b_{11}\right)\right) & \left(\underline{g}\left(b_{12}\right), \bar{g}\left(b_{12}\right)\right) & \cdots & \left(\underline{g}\left(b_{1 h}\right), \bar{g}\left(b_{1 h}\right)\right) \\
\left(\underline{g}\left(b_{21}\right), \bar{g}\left(b_{21}\right)\right) & \left(\underline{g}\left(b_{22}\right), \bar{g}\left(b_{22}\right)\right) & \cdots & \left(\underline{g}\left(b_{2 h}\right), \bar{g}\left(b_{2 h}\right)\right) \\
\left(\underline{g}\left(b_{31}\right), \bar{g}\left(b_{31}\right)\right) & \left(\underline{g}\left(b_{32}\right), \bar{g}\left(b_{32}\right)\right) & \cdots & \left(\underline{g}\left(b_{3 h}\right), \bar{g}\left(b_{3 h}\right)\right) \\
\vdots & \vdots & \ddots & \vdots \\
\left(\underline{g}\left(b_{g 1}\right), \bar{g}\left(b_{g 1}\right)\right) & \left(\underline{g}\left(b_{g 2}\right), \bar{g}\left(b_{g 2}\right)\right) & \cdots & \left(\underline{g}\left(b_{g h}\right), \bar{g}\left(b_{g h}\right)\right)
\end{array}\right],
$$

where the pair $(\underline{g}(b), \bar{g}(b))=\left\{\left(\xi_{\underline{g}(b)}(s), \eta_{\underline{g}(b)}(s), \psi_{\underline{g}(b)}(s)\right)\right.$, $\left.\left(\xi_{\bar{g}(b)}(s), \eta_{\bar{g}(b)}(s), \psi_{\bar{g}(b)}\right)\right\} \quad$ such that $0 \leq\left(\xi_{\bar{g}(b)}(s)\right)^{2}+$ $\left(\eta_{\bar{g}(b)}(s)\right)^{2}+\left(\psi_{\bar{g}(b)}(s)\right)^{2} \leq 1 \quad$ and $\quad 0 \leq\left(\xi_{\underline{g}(b)}(s)\right)^{2}+$ $\left(\eta_{\underline{g}(b)}(s)\right)^{2}+\left(\psi_{\underline{g}(b)}(s)\right)^{2} \leq 1$.
Step 1: construct the experts evaluation matrices $\mathscr{M}^{j}$ as follows:

$$
\mathscr{M}^{j}=\left[\begin{array}{cccc}
\left(\underline{g}\left(b_{11}^{j}\right), \bar{g}\left(b_{11}^{j}\right)\right) & \left(\underline{g}\left(b_{12}^{j}\right), \bar{g}\left(b_{12}^{j}\right)\right) & \ldots & \left(\underline{g}\left(b_{1 h}^{j}\right), \bar{g}\left(b_{1 h}^{j}\right)\right) \\
\left(\underline{g}\left(b_{21}^{j}\right), \bar{g}\left(b_{21}^{j}\right)\right) & \left(\underline{g}\left(b_{22}^{j}\right), \bar{g}\left(b_{22}^{j}\right)\right) & \ldots & \left(\underline{g}\left(b_{2 h}^{j}\right), \bar{g}\left(b_{2 h}^{j}\right)\right) \\
\left(\underline{g}\left(b_{31}^{j}\right), \bar{g}\left(b_{31}^{j}\right)\right) & \left(\underline{g}\left(b_{32}^{j}\right), \bar{g}\left(b_{32}^{j}\right)\right) & \ldots & \left(\underline{g}\left(b_{3 h}^{j}\right), \bar{g}\left(b_{3 h}^{j}\right)\right) \\
\vdots & \vdots & \ddots & \vdots \\
\left(\underline{g}\left(b_{g 1}^{j}\right), \bar{g}\left(b_{g 1}^{j}\right)\right) & \left(\underline{g}\left(b_{g 2}^{j}\right), \bar{g}\left(b_{g 2}^{j}\right)\right) & \ldots & \left(\underline{g}\left(b_{g h}^{j}\right), \bar{g}\left(b_{g h}^{j}\right)\right)
\end{array}\right],
$$

where $j$ represents the number of considered decision makers/experts.

Step 2: evaluate normalized experts' matrices $\mathscr{N}^{j}$ that is

$$
\mathscr{N}^{j}= \begin{cases}(\underline{g}(b), \bar{g}(b)), & \text { for benefit attributes, } \\ \left(\underline{g}(b)^{c}, \bar{g}(b)^{c}\right), & \text { for cost attributes. }\end{cases}
$$


Step 3: compute the collective performance of the experts using spherical fuzzy rough weighted averaging aggregation operator.

$$
\begin{aligned}
& \operatorname{SFRWA}\left(g\left(b_{1}\right), g\left(b_{1}\right), \ldots, g\left(b_{n}\right)\right)=\left\{\oplus_{i=1}^{n} u_{i} \underline{g}\left(b_{i}\right), \oplus_{i=1}^{n} u_{i} \bar{g}\left(b_{i}\right)\right\}
\end{aligned}
$$

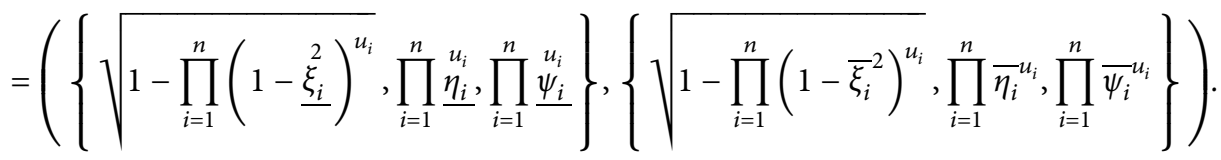

Step 4: utilizing the list of proposed aggregation operators, compute the aggregated spherical fuzzy rough information.

Step 5: compute the score (according to Definition 10) of aggregated alternatives.

Step 6: according to Definition 10, rank the alternatives $\left\{\beth_{1}, \beth_{2}, \ldots, \beth_{g}\right\}$ and choose the optimal one that has the higher value.

5.1. Numerical Application. A practical problem concerning decisions to evaluate industrial control system (ICS) security suppliers and select the appropriate one is considered as an example in this section to validate the applicability and practicality of the developed methodology.

5.2. Real Life Case Study. An ICS is a large class of automation systems used in manufacturing and industrial facilities to provide monitoring and control functions. Since more industrial control systems are connected to information technology (IT) networks and more IT technologies are implemented to industrial control systems to improve production efficiency, network security has emerged as a significant challenge. An ICS actually is the aggregate of a variety of system which is a vital part of national infrastructure such as electric power, transportation, energy, aviation and aerospace, and so on. To protect these systems from attack while maintaining normal operations, ICSaware control is essential. More than $80 \%$ of vital infrastructure relevant to people's livelihoods must depend on ICS to operate automatically. With the advancement of technology, ICS is increasingly facing more new threats like as viruses, Trojans, and hackers. The scale level of ICS security manufacturers is currently irregular. Many ICS security manufacturers also provide security systems that protect individual parts or groups of components, such as the industrial firewall, industrial gate, and so on. The difference between ICS and traditional information security is that ICS needs more necessary security rather than the impregnability of a single point of protection. To ensure comprehensive safety protection for ICS, an integrated safety system is required that can run throughout the entire manufacturing life cycle. Industrial control network security analysis and decision-making is an effective approach for resolving the problem because it can predict risks and help to make decisions before the fault of the industrial control network system. Therefore, it is essential for industrial enterprises to evaluate the entire capability of ICS security providers from various perspectives in order to select the best supplier finally. A company must now select one of four ICS security suppliers $\lambda_{i}(i=1,2,3,4)$ for better coordination. In addition, the invited decision makers are divided into three expert panels whose weighting vector is $\{0.19$, $0.35,0.46\}$ decided to evaluate the comprehensive capabilities of these four suppliers from the following four aspects:

(1) $\mathfrak{J}_{1}$ is the safety prevention and control capacity.

(2) $\mathfrak{\Im}_{2}$ is the product fit

(3) $\mathfrak{\Im}_{3}$ is the abnormal response time

(4) $\mathfrak{I}_{4}$ is future development capability of the enterprise Expert Information $=\left\{(E)^{1},(E)^{2},(E)^{3}\right\}$, where each expert panel is required to provide unified evaluation results in the form of q-rung orthopair fuzzy rough values with unknown expert and criteria weight information.

Step 1. The expert evaluation matrices under spherical fuzzy rough values are enclosed in Tables 2-4:

Step 2. The normalized experts' matrices $\mathcal{N}^{j}$ are enclosed in Tables 5-7:

Step 3. The collective performance of the experts using spherical fuzzy rough weighted averaging aggregation operator is enclosed in Table 8:

Step 4. In this step, we calculate the aggregated preference values of each alternative in the revised expert ideal matrix using the proposed list of aggregation operators as follows:

Case 1. Using SFRWA aggregation operator: The aggregated preference values of each alternative using SFRWA aggregation operator are enclosed in Table 9:

Case 2. Using SFROWA aggregation operator: The aggregated preference values of each alternative using SFROWA aggregation operator is enclosed in Table 10:

Case 3. Using SFRHWA aggregation operator: The aggregated preference values of each alternative using SFRHWA aggregation operator is evaluated as follows: 
TABLE 2: Expert-1 information.

\begin{tabular}{ccccc}
\hline & $\mathfrak{J}_{1}$ & $\mathfrak{\Im}_{2}$ & $\mathfrak{J}_{3}$ & $\mathfrak{J}_{4}$ \\
\hline$\lambda_{1}$ & $((0.84,0.34,0.40),(0.50,0.80$, & $((0.43,0.39,0.78),(0.46,0.23$, & $((0.67,0.50,0.30),(0.82,0.12$, & $((0.31,0.21,0.71),(0.55,0.21$, \\
& $0.30))$ & $0.51))$ & $0.43))$ & $0.63))$ \\
$\lambda_{2}$ & $((0.60,0.11,0.53),(0.88,0.23$, & $((0.11,0.21,0.91),(0.48,0.49$, & $((0.72,0.31,0.41),(0.73,0.13$, & $((0.11,0.25,0.82),(0.19,0.39$, \\
& $0.14))$ & $0.56))$ & $0.46))$ & $0.88))$ \\
$\lambda_{3}$ & $((0.79,0.19,0.39),(0.78,0.38$, & $((0.11,0.21,0.91),(0.48,0.49$, & $((0.71,0.41,0.13),(0.94,0.04$, & $((0.34,0.25,0.51),(0.39,0.19$, \\
& $0.18))$ & $0.56))$ & $0.07))$ & $0.61))$ \\
$\lambda_{4}$ & $((0.63,0.51,0.13),(0.83,0.39$, & $((0.49,0.33,0.42),(0.29,0.39$, & $((0.61,0.43,0.45),(0.85$, & $((0.49,0.37,0.59),(0.14,0.23$, \\
& $0.29))$ & $0.83))$ & $0.25 .0 .15))$ & $0.88))$ \\
\hline
\end{tabular}

TABle 3: Expert-2 information.

\begin{tabular}{ccccc}
\hline & $\mathfrak{J}_{1}$ & $\mathfrak{\Im}_{2}$ & $\mathfrak{\Im}_{3}$ & $\mathfrak{\Im}_{4}$ \\
\hline$\lambda_{1}$ & $((0.61,0.15,0.53),(0.59,0.37$, & $((0.16,0.35,0.62),(0.45,0.43$, & $((0.61,0.35,0.47),(0.42,0.33$, & $((0.55,0.17,0.74),(0.29,0.39$, \\
& $0.49))$ & $0.61))$ & $0.49))$ & $0.83))$ \\
$\lambda_{2}$ & $((0.66,0.11,0.51),(0.51,0.25$, & $((0.43,0.23,0.77),(0.13,0.41$, & $((0.93,0.08,0.09),(0.91,0.21$, & $((0.02,0.06,0.99),(0.48,0.49$, \\
& $0.34))$ & $0.11))$ & $0.56))$ \\
$\lambda_{3}$ & $((0.88,0.09,0.07),(0.82,0.25$, & $((0.05,0.06,0.89),(0.41,0.31$, & $((0.56,0.21,0.11),(0.59,0.35$, & $((0.43,0.13,0.61),(0.41,0.13$, \\
& $0.11))$ & $0.72))$ & $0.23))$ & $0.81))$ \\
$\lambda_{4}$ & $((0.59,0.32,0.34),(0.71,0.21$, & $((0.24,0.48,0.51),(0.30,0.50$, & $((0.68,0.53,0.39),(0.78,0.39$, & $((0.34,0.21,0.61),(0.46,0.23$, \\
& $0.31))$ & $0.67))$ & $0.43))$ & $0.51))$
\end{tabular}

TABLE 4: Expert-3 information.

\begin{tabular}{ccccc}
\hline & $\Im_{1}$ & $\Im_{2}$ & $\Im_{3}$ & $\Im_{4}$ \\
\hline$\lambda_{1}$ & $((0.85,0.25 .0 .15),(0.67,0.50$, & $((0.14,0.23,0.88),(0.42,0.33$, & $((0.78,0.38,0.18),(0.51,0.48$, & $((0.29,0.39,0.83),(0.34,0.21$, \\
& $0.30))$ & $0.49))$ & $0.24))$ & $0.61))$ \\
$\lambda_{2}$ & $((0.94,0.04,0.07),(0.72,0.31$, & $((0.39,0.19,0.61),(0.11,0.21$, & $((0.63,0.18,0.35),(0.89,0.06$, & $((0.48,0.49,0.56),(0.43,0.13$, \\
& $0.41))$ & $0.91))$ & $0.05))$ & $0.61))$ \\
$\lambda_{3}$ & $((0.73,0.13,0.46),(0.71,0.41$, & $((0.19,0.39,0.88),(0.23,0.35$, & $((0.87,0.35,0.18),(0.77,0.23$, & $((0.41,0.13,0.81),(0.02,0.06$, \\
& $0.13))$ & $0.59))$ & $0.43))$ & $0.99))$ \\
$\lambda_{4}$ & $((0.82,0.12,0.43),(0.61,0.43$, & $((0.55,0.21,0.63),(0.43,0.39$, & $((0.53,0.33,0.47),(0.62,0.35$, & $((0.46,0.23,0.51),(0.55,0.17$, \\
& $0.45))$ & $0.78))$ & $0.16))$ & $0.74))$
\end{tabular}

TABle 5: Normalized Expert-1 information.

\begin{tabular}{ccccc}
\hline & $\mathfrak{J}_{1}$ & $\mathfrak{\Im}_{2}$ & $\Im_{3}$ & $\Im_{4}$ \\
\hline$\lambda_{1}$ & $((0.84,0.34,0.40),(0.50,0.80$, & $((0.78,0.39,0.43),(0.51,0.23$, & $((0.67,0.50,0.30),(0.82,0.12$, & $((0.71,0.21,0.31),(0.63,0.21$, \\
& $0.30))$ & $0.46))$ & $0.43))$ & $0.55))$ \\
$\lambda_{2}$ & $((0.60,0.11,0.53),(0.88,0.23$, & $((0.59,0.35,0.23),(0.81,0.13$, & $((0.72,0.31,0.41),(0.73,0.13$, & $((0.82,0.25,0.11),(0.88,0.39$, \\
& $0.14))$ & $0.41))$ & $0.46))$ & $0.19))$ \\
$\lambda_{3}$ & $((0.79,0.19,0.39),(0.78,0.38$, & $((0.91,0.21,0.11),(0.56,0.49$, & $((0.71,0.41,0.13),(0.94,0.04$, & $((0.51,0.25,0.34),(0.61,0.19$, \\
& $0.18))$ & $0.48))$ & $0.07))$ & $0.39))$ \\
$\lambda_{4}$ & $((0.63,0.51,0.13),(0.83,0.39$, & $((0.42,0.33,0.49),(0.83,0.39$, & $((0.61,0.43,0.45),(0.85$, & $((0.59,0.37,0.49),(0.88,0.23$, \\
& $0.29))$ & $0.29))$ & $0.25 .0 .15))$ & $0.14))$ \\
\hline
\end{tabular}

TABLE 6: Normalized Expert-2 information.

\begin{tabular}{ccccc}
\hline & $\mathfrak{J}_{1}$ & $\mathfrak{\Im}_{2}$ & $\mathfrak{J}_{3}$ & $\mathfrak{J}_{4}$ \\
\hline$\lambda_{1}$ & $((0.61,0.15,0.53),(0.59,0.37$, & $((0.62,0.35,0.16),(0.61,0.43$, & $((0.61,0.35,0.47),(0.42,0.33$, & $((0.74,0.17,0.55),(0.83,0.39$, \\
& $0.49))$ & $0.45))$ & $0.49))$ & $0.29))$ \\
$\lambda_{2}$ & $((0.66,0.11,0.51),(0.51,0.25$, & $((0.77,0.23,0.43),(0.71,0.41$, & $((0.93,0.08,0.09),(0.91,0.21$, & $((0.99,0.06,0.02),(0.56,0.49$, \\
& $0.34))$ & $0.13))$ & $0.11))$ & $0.48))$ \\
$\lambda_{3}$ & $((0.88,0.09,0.07),(0.82,0.25$, & $((0.89,0.06,0.05),(0.72,0.31$, & $((0.56,0.17,0.44),(0.59,0.35$, & $((0.61,0.13,0.43),(0.81,0.13$, \\
& $0.11))$ & $0.41))$ & $0.23))$ & $0.41))$ \\
$\lambda_{4}$ & $((0.59,0.32,0.34),(0.71,0.21$, & $((0.51,0.48,0.24),(0.67,0.50$, & $((0.68,0.53,0.39),(0.78,0.39$, & $((0.61,0.21,0.34),(0.51,0.23$, \\
& $0.31))$ & $0.30))$ & $0.43))$ & $0.46))$
\end{tabular}


TABLE 7: Normalized Expert-3 information.

\begin{tabular}{ccccc}
\hline & $\mathfrak{J}_{1}$ & $\mathfrak{J}_{2}$ & $\mathfrak{I}_{3}$ & $\mathfrak{I}_{4}$ \\
\hline$\lambda_{1}$ & $((0.85,0.25,0.15),(0.67,0.50$, & $((0.88,0.23,0.14),(0.49,0.33$, & $((0.78,0.38,0.18),(0.51,0.48$, & $((0.83,0.39,0.29),(0.61,0.21$, \\
& $0.30))$ & $0.42))$ & $0.24))$ & $0.34))$ \\
$\lambda_{2}$ & $((0.94,0.04,0.07),(0.72,0.31$, & $((0.61,0.19,0.39),(0.91,0.21$, & $((0.63,0.18,0.35),(0.89,0.06$, & $((0.56,0.49,0.48),(0.61,0.13$, \\
& $0.41))$ & $0.05))$ & $0.43))$ \\
$\lambda_{3}$ & $((0.73,0.13,0.46),(0.71,0.41$, & $((0.88,0.39,0.19),(0.59,0.35$, & $((0.87,0.35,0.18),(0.77,0.23$, & $((0.81,0.13,0.41),(0.99,0.06$, \\
& $0.13))$ & $0.23))$ & $0.43))$ & $0.02))$ \\
$\lambda_{4}$ & $((0.82,0.12,0.43),(0.61,0.43$, & $((0.63,0.21,0.55),(0.78,0.39$, & $((0.53,0.33,0.47),(0.62,0.35$, & $((0.51,0.23,0.46),(0.74,0.17$, \\
& $0.45))$ & $0.43))$ & $0.16))$ & $0.55))$ \\
\hline
\end{tabular}

TABLE 8: Experts collective matrix.

\begin{tabular}{ccccc}
\hline & $\mathfrak{\Im}_{1}$ & $\mathfrak{\Im}_{2}$ & $\Im_{3}$ & $\Im_{4}$ \\
\hline$\lambda_{1}$ & $((0.78,0.22,0.31),(0.59,0.52$, & $((0.78,0.31,0.20),(0.54,0.32$, & $((0.69,0.40,0.29),(0.63,0.27$, & $((0.76,0.23,0.37),(0.71,0.26$, \\
& $0.35))$ & $0.44))$ & $0.34))$ & $0.37))$ \\
$\lambda_{2}$ & $((0.80,0.07,0.27),(0.74,0.26$, & $((0.67,0.24,0.34),(0.82,0.22$, & $((0.81,0.16,0.22),(0.86,0.11$, & $((0.91,0.18,0.09),(0.72,0.29$, \\
& $0.27))$ & $0.17))$ & $0.13))$ & $0.34))$ \\
$\lambda_{3}$ & $((0.81,0.12,0.22),(0.77,0.33$, & $((0.89,0.16,0.09),(0.63,0.37$, & $((0.74,0.28,0.22),(0.83,0.15$, & $((0.67,0.15,0.39),(0.91,0.11$, \\
& $0.13))$ & $0.35))$ & $0.19))$ & $0.14))$ \\
$\lambda_{4}$ & $((0.70,0.26,0.27),(0.73,0.32$, & $((0.53,0.32,0.39),(0.76,0.42$, & $((0.61,0.42,0.43),(0.76,0.32$, & $((0.57,0.25,0.42),(0.75,0.20$, \\
& $0.34))$ & $0.33))$ & $0.22))$ & $0.33))$
\end{tabular}

TABLE 9: Aggregated information using SFRWA.

\begin{tabular}{ll}
\hline$\lambda_{1}$ & $((0.7559,0.2808,0.2865),(0.6312,0.3164,0.3763))$ \\
$\lambda_{2}$ & $((0.8260,0.1582,0.1950),(0.7923,0.2109,0.2163))$ \\
$\lambda_{3}$ & $((0.7937,0.1689,0.2064),(0.8211,0.2020,0.1897))$ \\
$\lambda_{4}$ & $((0.5997,0.3034,0.3806),(0.7515,0.2977,0.3023))$ \\
\hline
\end{tabular}

TABLe 10: Aggregated information using SFROWA.

\begin{tabular}{ll}
\hline$\lambda_{1}$ & $((0.7584,0.2793,0.2805),(0.6151,0.3439,0.3738))$ \\
$\lambda_{2}$ & $((0.8164,0.1549,0.2073),(0.7907,0.2159,0.2167))$ \\
$\lambda_{3}$ & $((0.8107,0.1663,0.1859),(0.7956,0.2315,0.1956))$ \\
$\lambda_{4}$ & $((0.5984,0.3064,0.3795),(0.7519,0.3067,0.3023))$ \\
\hline
\end{tabular}

For SFRHWA operator, firstly the weighted collected matrix is enclosed in Table 11:

Respected score value is evaluated in Table 12:

Ordered weighted metric by using score value is enclosed in Table 13:

The aggregated preference values of each alternative using SFRHWA aggregation operator is evaluated in Table 14:

Step 5. Score of aggregated overall preference values of each alternative is enclosed in Table 15:

Step 6. Rank of the alternatives $\lambda_{k}(k=1,2, \ldots, 4)$ is enclosed in Table 15:

From the above computational process, we concluded that alternative $\lambda_{3}$ is the best among others, and therefore it is highly recommended.

The ranking results of the alternative using proposed aggregation operators are shown in Figure 1.
5.3. Reliability and Validity Test. In practice, selecting the perfect option from the group's decision matrices is a challenging task. The approach for analyzing the reliability and validity of DM systems was developed by Wang and Triantaphyllou [52]. The testing procedure is as follows.

Test Step 1: the appropriate and effective MAGDM technique is to present the appropriate choice without modification and without changing the comparable position of each decision criterion by substituting the normalized element for the worse element of the alternative.

Test Step 2: the transitive property must be satisfied using an efficient and appropriate MAGDM approach.

Test Step 3: when a MAGDM problem is reduced to a minor one. A combined alternative rating should be similar to the original rating of the un-decomposed problem. To rank the alternative, we utilize the same 
TABLE 11: Weighted collected matrix.

\begin{tabular}{lccc}
\hline \multicolumn{1}{c}{$\mathfrak{\Im}_{1}$} & $\mathfrak{\Im}_{2}$ & $\mathfrak{\Im}_{3}$ & $\Im_{4}$ \\
\hline$(0.4039,0.7500,0.8005$, & $(0.4729,0.7289$, & $(0.3717,0.8100,0.7522,0.3314$, & $(0.4842,0.6341,0.7348,0.4421$, \\
$0.2794,0.8832,0.8192)$ & $0.6476,0.2981,0.7352,0.8012)$ & $0.7400,0.7803)$ & $0.6586,0.7348)$ \\
$(0.4200,0.6033,0.7798,0.3740$, & $(0.3855,0.6802$, & $(0.4666,0.6561,0.7059,0.5159$, & $(0.6486,0.5877,0.4740,0.4502$, \\
$0.7742,0.7798)$ & $0.7473,0.5101,0.6644,0.6198)$ & $0.6019,0.6255)$ & $0.6813,0.7157)$ \\
$(0.4284,0.6684,0.7500,0.3962$, & $(0.5879,0.6097$, & $(0.4084,0.7462,0.7059,0.4853$, & $(0.4107,0.5554,0.7468,0.6486$, \\
$0.8101,0.6787)$ & $0.5220,0.3572,0.7646,0.7532)$ & $0.6464,0.6825)$ & $0.5045,0.5436)$ \\
$(0.3465,0.7742,0.7798$, & $(0.2919,0.7289,0.7755,0.4556$, & $(0.3186,0.8191,0.8236,0.4240$, & $(0.3386,0.6507,0.7642,0.4755$, \\
$0.3670,0.8053,0.8147)$ & $0.7352,0.7413)$ & $0.7695,0.7059)$ & $0.6072,0.7092)$ \\
\hline
\end{tabular}

TABLE 12: Score of weighted matrix.

\begin{tabular}{lcccr}
\hline & $\mathfrak{I}_{1}$ & $\mathfrak{I}_{2}$ & $\mathfrak{\Im}_{3}$ & $\mathfrak{I}_{4}$ \\
\hline$\lambda_{1}$ & 0.2384 & 0.3097 & 0.2701 & 0.3607 \\
$\lambda_{2}$ & 0.3095 & 0.3640 & 0.3988 & 0.4400 \\
$\lambda_{3}$ & 0.3196 & 0.3826 & 0.3521 & 0.4515 \\
$\lambda_{4}$ & 0.2566 & 0.2944 & 0.2708 & 0.3471 \\
\hline
\end{tabular}

TABle 13: Ordered weighted collected matrix.

\begin{tabular}{lccc}
\hline $\mathfrak{I}_{1}$ & $\mathfrak{\Im}_{2}$ & $\mathfrak{\Im}_{3}$ & $\mathfrak{\Im}_{4}$ \\
\hline$(0.4842,0.6341,0.7348,0.4421$, & $(0.4729,0.7289,0.6476,0.2981$, & $(0.3717,0.8100,0.7522,0.3314$, & $(0.4039,0.7500,0.8005,0.2794$, \\
$0.6586,0.7348)$ & $0.7352,0.8012)$ & $0.7400,0.7803)$ & $0.8832,0.8192)$ \\
$(0.6486,0.5877,0.4740,0.4502$, & $(0.4666,0.6561,0.7059,0.5159$, & $(0.3855,0.6802,0.7473,0.5101$, & $(0.4200,0.6033,0.7798,0.3740$, \\
$0.6813,0.7157)$ & $0.6019,0.6255)$ & $0.6644,0.6198)$ & $0.7742,0.7798)$ \\
$(0.4107,0.5554,0.7468,0.6486$, & $(0.5879,0.6097,0.5220,0.3572$, & $(0.4084,0.7462,0.7059,0.4853$, & $(0.4284,0.6684,0.7500,0.3962$, \\
$0.5045,0.5436)$ & $0.7646,0.7532)$ & $0.6464,0.6825)$ & $0.8101,0.6787)$ \\
$(0.3386,0.6507,0.7642,0.4755$, & $(0.2919,0.7289,0.7755,0.4556$, & $(0.3186,0.8191,0.8236,0.4240$, & $(0.3465,0.7742,0.7798,0.3670$, \\
$0.6072,0.7092)$ & $0.7352,0.7413)$ & $0.7695,0.7059)$ & $0.8053,0.8147)$ \\
\hline
\end{tabular}

TABLe 14: Aggregated information using SFRHWA.

\begin{tabular}{ll}
\hline$\lambda_{1}$ & $((0.4338,0.7338,0.7332),(0.3342,0.7633,0.7888))$ \\
$\lambda_{2}$ & $((0.4842,0.6312,0.6839),(0.4641,0.6816,0.6857))$ \\
$\lambda_{3}$ & $((0.4740,0.6456,0.6701),(0.4747,0.6920,0.6701))$ \\
$\lambda_{4}$ & $((0.3248,0.7466,0.7855),(0.4276,0.7369,0.7485))$ \\
\hline
\end{tabular}

TABLE 15: Ranking of alternative.

\begin{tabular}{lccccc}
\hline \multirow{2}{*}{ Proposed operators } & \multicolumn{2}{c}{ Score values of alternatives } & $\lambda_{4}$ & \multicolumn{2}{c}{ Ranking } \\
& $\lambda_{1}$ & $\lambda_{2}$ & $\lambda_{3}$ & 0.6779 & $\lambda_{3}>\lambda_{2}>\lambda_{1}>\lambda_{4}$ \\
SFRWA & 0.6878 & 0.8063 & 0.8080 & 0.6759 & $\lambda_{3}>\lambda_{2}>\lambda_{1}>\lambda_{4}$ \\
SFROWA & 0.6827 & 0.8020 & 0.8045 & 0.2892 & $\lambda_{3}>\lambda_{2}>\lambda_{1}>\lambda_{4}$ \\
SFRHWA & 0.2915 & 0.3777 & 0.3785 & 0.25 \\
\hline
\end{tabular}

methods adopted in the MAGDM problem on minor issues.

The MAGDM problem was scaled down to achieve the best results, and the same suggested DM technique was applied. If the same procedure is applied to a small problem, the result will be the same as the MAGDM problem.
5.4. Validity Test (Proposed Numerical Illustration). The collected expert information in proposed case study is enclosed in Table 8:

Test Step 1: we replace the worst component of the alternative by presenting the effective choice without a modification and also without changing the 


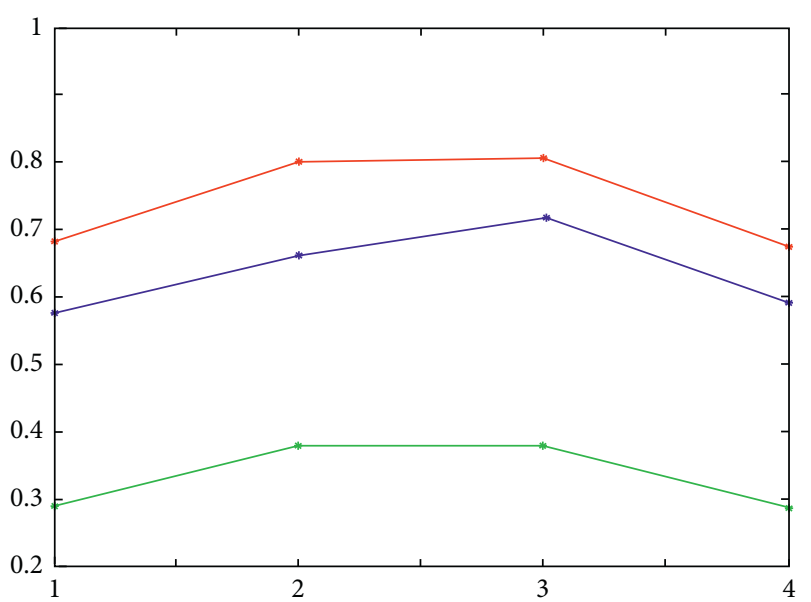

Figure 1: Graphical representation of alternatives using EWA, EOWA and EHWA operators.

comparable position of each decision criterion. Table 16 contains the most recent expert decision matrix compilation.

Case 1: using SFRWA aggregation operator: the aggregated preference values of each alternative in the updated collected expert's decision matrix using SFRWA aggregation operator are enclosed in Table 17:

Case 2: using SFROWA aggregation operator: the aggregated preference values of each alternative in the updated collected expert's decision matrix using SFROWA aggregation operator are enclosed in Table 18:
Rank of the alternatives $\lambda_{k}(k=1,2, \ldots, 4)$ is enclosed in Table 19:

When we use Test Step 1, we get the same result $\lambda_{3}$, which is also the case when we use our established method.

The ranking results of the alternative in the validity test under proposed aggregation operators are shown in Figure 2.

Test Step 2 and 3: Steps 2 and 3 of the validity tests is now being tested to indicate that the presented method is reliable and valid. To accomplish this, we first divided the MAGDM problem into three smaller sub-problems, such as $\left\{\lambda_{3}, \lambda_{2}, \lambda_{1}\right\},\left\{\lambda_{2}, \lambda_{1}, \lambda_{4}\right\}$, and $\left\{\lambda_{3}, \lambda_{1}, \lambda_{4}\right\}$. We now implement our presented DM approach to the smaller problems that have been converted and give us the ranking of alternatives as: $\lambda_{3}>\lambda_{2}>\lambda, \lambda_{2}>\lambda_{1}>\lambda_{4}$ and $\lambda_{3}>\lambda_{1}>\lambda_{4}$ respectively. We analyzed that $\lambda_{3}>\lambda_{2}>\lambda_{1}>\lambda_{4}$ is the same as the standard decision-making approach results when assigning a detailed ranking.

\section{TOPSIS Methodology}

We present a strategy for dealing with uncertainty in DM under the presence of SFR information. Consider a DM problem with $\left.\left\{\beth_{1}, \beth_{2}, \ldots,\right\lrcorner_{g}\right\}$ be a set of alternative and $\left.\left\{\neg_{1}, \neg_{2}, \ldots,\right\urcorner_{h}\right\}$ be a set of attributes with $\left(\partial_{1}, \partial_{2}, \ldots, \partial_{h}\right)$ be the weight vector, such that each $\partial_{t} \epsilon[0,1], \sum_{t=1}^{n} \partial_{t}=1$. To test the reliability of kth alternative $\beth_{k}$ under the $t$ th attribute $\neg_{t}$, let a set of decision makers (DMs) $\left\{D_{1}, D_{2}, \ldots, D_{j}\right\}$ and $\left(\eta_{1}, \eta_{2}, \ldots, \eta_{j}\right)$ be DMs weights such that $\eta_{t} \epsilon[0,1], \sum_{t=1}^{j} \eta_{t}=1$. The expert evaluation matrix is described as follows:

$$
M=\left[g^{j}(b)\right]_{\mathbf{g} \times \mathbf{h}}=\left[\begin{array}{cccc}
\left(\underline{g}\left(b_{11}\right), \bar{g}\left(b_{11}\right)\right) & \left(\underline{g}\left(b_{12}\right), \bar{g}\left(b_{12}\right)\right) & \ldots & \left(\underline{g}\left(b_{1 h}\right), \bar{g}\left(b_{1 h}\right)\right) \\
\left(\underline{g}\left(b_{21}\right), \bar{g}\left(b_{21}\right)\right) & \left(\underline{g}\left(b_{22}\right), \bar{g}\left(b_{22}\right)\right) & \ldots & \left(\underline{g}\left(b_{2 h}\right), \bar{g}\left(b_{2 h}\right)\right) \\
\left(\underline{g}\left(b_{31}\right), \bar{g}\left(b_{31}\right)\right) & \left(\underline{g}\left(b_{32}\right), \bar{g}\left(b_{32}\right)\right) & \ldots & \left(\underline{g}\left(b_{3 h}\right), \bar{g}\left(b_{3 h}\right)\right) \\
\vdots & \vdots & \ddots & \vdots \\
\left(\underline{g}\left(b_{g 1}\right), \bar{g}\left(b_{g 1}\right)\right) & \left(\underline{g}\left(b_{g 2}\right), \bar{g}\left(b_{g 2}\right)\right) & \cdots & \left(\underline{g}\left(b_{g h}\right), \bar{g}\left(b_{g h}\right)\right)
\end{array}\right]
$$

where the pair $(\underline{g}(b), \bar{g}(b))=\left\{\left(\xi_{\underline{g}_{(b)}}(s), \eta_{\underline{g}(b)}(s), \psi_{\underline{g}(b)}(s)\right)\right.$, $\left.\left(\xi_{\bar{g}(b)}(s), \eta_{\bar{g}(b)}(s), \psi_{\bar{g}(b)}\right)\right\} \quad$ such that $0 \leq\left(\xi_{\bar{g}(b)}(s)\right)^{2}+$

$$
\left(\eta_{\bar{g}(b)}(s)\right)^{2}+\left(\psi_{\bar{g}(b)}(s)\right)^{2} \leq 1 \quad \text { and } \quad 0 \leq\left(\xi_{\underline{g}(b)}(s)\right)^{2}+
$$$$
\left(\eta_{\underline{g}(b)}(s)\right)^{2}+\left(\psi_{\underline{g}(b)}(s)\right)^{2} \leq 1 .
$$

Step 1: construct the experts evaluation matrices $\mathscr{M}^{j}$.

$$
\mathscr{M}^{j}=\left[\begin{array}{cccc}
\left(\underline{g}\left(b_{11}^{j}\right), \bar{g}\left(b_{11}^{j}\right)\right) & \left(\underline{g}\left(b_{12}^{j}\right), \bar{g}\left(b_{12}^{j}\right)\right) & \cdots & \left(\underline{g}\left(b_{1 h}^{j}\right), \bar{g}\left(b_{1 h}^{j}\right)\right) \\
\left(\underline{g}\left(b_{21}^{j}\right), \bar{g}\left(b_{21}^{j}\right)\right) & \left(\underline{g}\left(b_{22}^{j}\right), \bar{g}\left(b_{22}^{j}\right)\right) & \cdots & \left(\underline{g}\left(b_{2 h}^{j}\right), \bar{g}\left(b_{2 h}^{j}\right)\right) \\
\left(\underline{g}\left(b_{31}^{j}\right), \bar{g}\left(b_{31}^{j}\right)\right) & \left(\underline{g}\left(b_{32}^{j}\right), \bar{g}\left(b_{32}^{j}\right)\right) & \cdots & \left(\underline{g}\left(b_{3 h}^{j}\right), \bar{g}\left(b_{3 h}^{j}\right)\right) \\
\vdots & \vdots & \ddots & \vdots \\
\left(\underline{g}\left(b_{g 1}^{j}\right), \bar{g}\left(b_{g 1}^{j}\right)\right) & \left(\underline{g}\left(b_{g 2}^{j}\right), \bar{g}\left(b_{g 2}^{j}\right)\right) & \cdots & \left(\underline{g}\left(b_{g h}^{j}\right), \bar{g}\left(b_{g h}^{j}\right)\right)
\end{array}\right],
$$


TABle 16: Updated Expert's collective matrix.

\begin{tabular}{ccccc}
\hline & $\mathfrak{J}_{1}$ & $\mathfrak{\Im}_{2}$ & $\mathfrak{J}_{3}$ & $\mathfrak{I}_{4}$ \\
\hline$\lambda_{1}$ & $((0.78,0.22,0.31),(0.59,0.52$, & $((0.20,0.31,0.78),(0.44,0.32$, & $((0.69,0.40,0.29),(0.63,0.27$, & $((0.37,0.23,0.76),(0.37,0.26$, \\
& $0.35))$ & $0.54))$ & $0.34))$ & $0.71))$ \\
$\lambda_{2}$ & $((0.80,0.07,0.27),(0.74,0.26$, & $((0.34,0.24,0.67),(0.17,0.22$, & $((0.81,0.16,0.22),(0.86,0.11$, & $((0.09,0.18,0.91),(0.34,0.29$, \\
& $0.27))$ & $0.13))$ & $0.72))$ \\
$\lambda_{3}$ & $((0.22,0.12,0.81),(0.13,0.33$, & $((0.89,0.16,0.09),(0.63,0.37$, & $((0.22,0.28,0.74),(0.19,0.15$, & $((0.67,0.15,0.39),(0.91,0.11$, \\
& $0.77))$ & $0.35))$ & $0.83))$ & $0.14))$ \\
$\lambda_{4}$ & $((0.27,0.26,0.70),(0.34,0.32$, & $((0.53,0.32,0.39),(0.76,0.42$, & $((0.43,0.42,0.61),(0.22,0.32$, & $((0.57,0.25,0.42),(0.75,0.20$, \\
& $0.73))$ & $0.33))$ & $0.76))$ & $0.33))$ \\
\hline
\end{tabular}

TABLE 17: Aggregated information using SFRWA.

\begin{tabular}{ll}
\hline$\lambda_{1}$ & $((0.5644,0.2808,0.5172),(0.5101,0.3164,0.4867))$ \\
$\lambda_{2}$ & $((0.6151,0.1582,0.4798),(0.6302,0.2109,0.4175))$ \\
$\lambda_{3}$ & $((0.6835,0.1689,0.3495),(0.7074,0.2020,0.3733))$ \\
$\lambda_{4}$ & $((0.4882,0.3034,0.4943),(0.6385,0.2977,0.4649))$ \\
\hline
\end{tabular}

TABLE 18: Aggregated information using SFROWA.

\begin{tabular}{lr}
\hline$\lambda_{1}$ & $((0.5465,0.2793,0.5392),(0.5422,0.3439,0.4644))$ \\
$\lambda_{2}$ & $((0.6165,0.1549,0.4779),(0.6176,0.2159,0.4321))$ \\
$\lambda_{3}$ & $((0.7474,0.1663,0.2724),(0.5809,0.2315,0.3918))$ \\
$\lambda_{4}$ & $((0.4862,0.3064,0.4928),(0.6391,0.3067,0.4649))$ \\
\hline
\end{tabular}

TABLE 19: Ranking of alternative.

\begin{tabular}{lccccc}
\hline \multirow{2}{*}{ Proposed operators } & \multicolumn{2}{c}{ Score values of alternatives } & $\lambda_{4}$ & Ranking \\
& $\lambda_{1}$ & $\lambda_{2}$ & $\lambda_{3}$ & 0.5944 & $\lambda_{3}>\lambda_{2}>\lambda_{1}>\lambda_{4}$ \\
SFRWA-test & 0.5789 & 0.6631 & 0.7162 & 0.5924 & $\lambda_{3}>\lambda_{2}>\lambda_{1}>\lambda_{4}$ \\
SFROWA-test & 0.5770 & 0.6589 & 0.7111 &
\end{tabular}

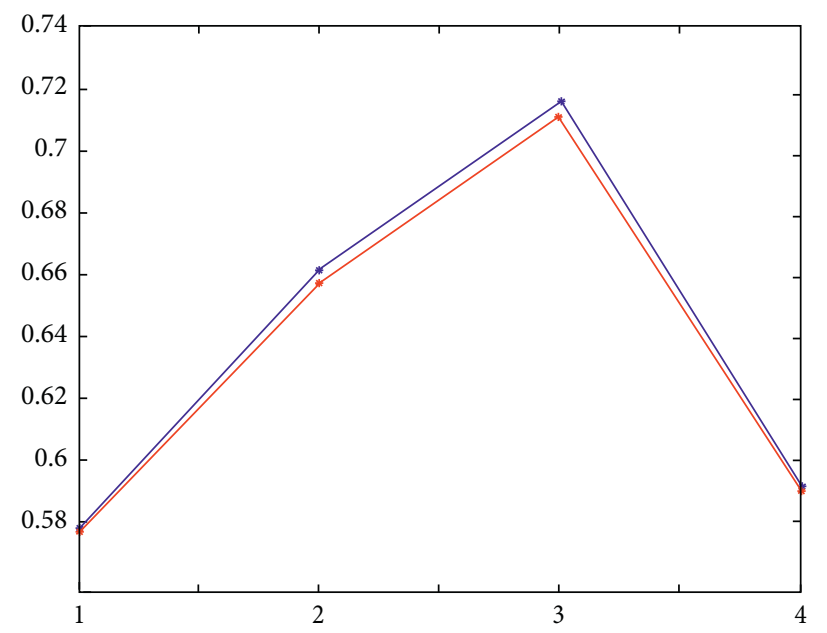

FIGURE 2: Graphical representation of alternatives using test EWA and EOWA operators. 
where $j$ represents the number of considered decision makers/experts.

Step 2: evaluate normalized experts' matrices $\mathscr{N}^{j}$ that is $\mathscr{N}^{j}= \begin{cases}(\underline{g}(b), \bar{g}(v)), & \text { for benefit attributes, } \\ \left(\underline{g}(b)^{c}, \bar{g}(v)^{c}\right), & \text { for cost attributes. }\end{cases}$
Step 3: compute the collective performance of the experts using spherical fuzzy rough weighted averaging aggregation operator.

$$
\begin{aligned}
& \operatorname{SFRWA}\left(g\left(b_{1}\right), g\left(b_{1}\right), \ldots, g\left(b_{n}\right)\right)=\left\{\oplus_{i=1}^{n} u_{i} \underline{g}\left(b_{i}\right), \oplus_{i=1}^{n} u_{i} \bar{g}\left(b_{i}\right),\right\}
\end{aligned}
$$

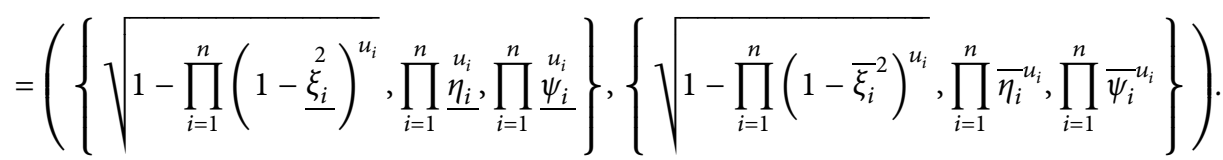

Step 4: determine the positive and negative ideal solutions $I^{+}$and $I^{-}$for DM's by using normalized decision matrix are defined as follows:

$$
\begin{array}{ll}
I^{+}=\left\{\left(N^{(j)}\right):\right. & \left.\underset{\max }{i}\left[S C\left(N_{i j}^{(l)}\right)\right]\right\}, \quad(j=1,2, \ldots, n), \\
I^{-}=\left\{\left(N^{(j)}\right):\right. & \left.{ }_{\min }^{i}\left[S C\left(N_{i j}^{(l)}\right)\right]\right\}, \quad(j=1,2, \ldots, n) .
\end{array}
$$

Step 5: by using equation (14) the WGDM from $D M\left(N^{*}\right)^{(l)}$ to $P^{*} I S^{(l)}$ and $N^{*} I S^{(l)}$, are computed as follows:

$$
\begin{aligned}
& D I S_{i}^{+(l)}=\left(\frac{1}{4 n} \sum_{j=1}^{n} c_{j}\left(\begin{array}{l}
\left|\left(\underline{\mu}_{D M\left(N^{*}\right)^{(l)}}\right)^{q}-\left(\underline{\mu}_{P^{*} I S^{(l)}}\right)^{q}\right|^{\lambda}+\left|\left(\underline{\nu}_{D M\left(N^{*}\right)^{(l)}}\right)^{q}-\left(\underline{\nu}_{P^{*} I S^{(l)}}\right)^{q}\right|^{\lambda} \\
\left|\left(\bar{\mu}_{D M\left(N^{*}\right)^{(l)}}\right)^{q}-\left(\bar{\mu}_{P^{*} I S^{(l)}}\right)^{q}\right|^{\lambda}+\left|\left(\bar{\nu}_{D M\left(N^{*}\right)^{(l)}}\right)^{q}-\left(\bar{\nu}_{P^{*} I S^{(l)}}\right)^{q}\right|^{\lambda}+
\end{array}\right)\right)^{1 / \lambda} \\
& D I S_{i}^{-(l)}=\left(\frac{1}{4 n} \sum_{j=1}^{n} c_{j}\left(\begin{array}{l}
\left|\left(\underline{\mu}_{D M\left(N^{*}\right)^{(l)}}\right)^{q}-\left(\underline{\mu}_{N^{*} I S^{(l)}}\right)^{q}\right|^{\lambda}+\left|\left(\underline{v}_{D M\left(N^{*}\right)^{(l)}}\right)^{q}-\left(\underline{v}_{N^{*} I S^{(l)}}\right)^{q}\right|^{\lambda} \\
\left|\left(\bar{\mu}_{D M\left(N^{*}\right)^{(l)}}\right)^{q}-\left(\bar{\mu}_{N^{*} I S^{(l)}}\right)^{q}\right|^{\lambda}+\left|\left(\bar{v}_{D M\left(N^{*}\right)^{(l)}}\right)^{q}-\left(\bar{\nu}_{N^{*} I S^{(l)}}\right)^{q}\right|^{\lambda}+
\end{array}\right)\right)^{1 / \lambda}
\end{aligned}
$$

and for each $i=1,2, \ldots, m$.

Step 6: the closeness indices denoted by $\left(\dot{C}^{*} I_{i}\right)$ are calculated as follows:

$$
\dot{C}^{*} I_{i}=\frac{\mathrm{DIS}_{i}^{-(l)}}{\mathrm{DIS}_{i}^{+(l)}+\mathrm{DIS}_{i}^{-(l)}} .
$$

In ascending order, rank the measured $\left(\dot{C}^{*} I s\right)$ values; the alternative with the greatest value is our best choice.

\subsection{Numerical Illustration}

Step 1: the expert evaluation matrices under spherical fuzzy rough values are enclosed in Tables $2-4$ which is given in real life case study.

Step 2: the normalized experts' matrices $\mathcal{N}^{j}$ is enclosed in Tables 5-7 which is given in real life case study.
Step 3: the collective performance of the experts using spherical fuzzy rough weighted averaging aggregation operator is enclosed in Table 20:

Step 4: the positive and negative ideal solutions $I^{+}$and $I^{-}$for DM's by using normalized decision matrix are evaluated in Table 21:

Step 5: by using equation (14) the WGDM from $D M\left(N^{*}\right)^{(l)}$ to $P^{*} I S^{(l)}$ and $N^{*} I S^{(l)}$, are computed as follows:

$$
\begin{array}{llll}
0.3327 & 0.2680 & 0.0302 & 0.3501 \\
0.2256 & 0.3442 & 0.3490 & 0.0651
\end{array}
$$

Step 6: the closeness indices denoted by $\left(\dot{C}^{*} I_{i}\right)$ are calculated in Table 22.

Ranking results using SFR-TOPSIS methodology is shown in Figure 3: 
TABLE 20: Experts collective matrix.

\begin{tabular}{ccccc}
\hline & $\mathfrak{J}_{1}$ & $\mathfrak{\Im}_{2}$ & $\Im_{3}$ & $\mathfrak{J}_{4}$ \\
\hline$\lambda_{1}$ & $((0.78,0.22,0.31),(0.59,0.52$, & $((0.78,0.31,0.20),(0.54,0.32$, & $((0.69,0.40,0.29),(0.63,0.27$, & $((0.76,0.23,0.37),(0.71,0.26$, \\
& $0.35))$ & $0.44))$ & $0.34))$ & $0.37))$ \\
$\lambda_{2}$ & $((0.80,0.07,0.27),(0.74,0.26$, & $((0.67,0.24,0.34),(0.82,0.22$, & $((0.81,0.16,0.22),(0.86,0.11$, & $((0.91,0.18,0.09),(0.72,0.29$, \\
& $0.27))$ & $0.13))$ & $0.34))$ \\
$\lambda_{3}$ & $((0.81,0.12,0.22),(0.77,0.33$, & $((0.89,0.16,0.09),(0.63,0.37$, & $((0.74,0.28,0.22),(0.83,0.15$, & $((0.67,0.15,0.39),(0.91,0.11$, \\
& $0.13))$ & $0.19))$ & $0.14))$ \\
$\lambda_{4}$ & $((0.70,0.26,0.27),(0.73,0.32$, & $((0.53,0.32,0.39),(0.76,0.42$, & $((0.61,0.42,0.43),(0.76,0.32$, & $((0.57,0.25,0.42),(0.75,0.20$, \\
& $0.34))$ & $0.33))$ & $0.22))$ & $0.33))$ \\
\hline
\end{tabular}

TABLE 21: Ideal solution.

\begin{tabular}{lcc}
\hline & $\mathrm{I}^{+}$ & $\mathrm{I}^{-}$ \\
\hline$\lambda_{1}$ & $((0.81,0.12,0.22),(0.77,0.33,0.13))$ & $((0.78,0.22,0.31),(0.59,0.52,0.35))$ \\
$\lambda_{2}$ & $((0.89,0.16,0.09),(0.63,0.37,0.35))$ & $((0.53,0.32,0.39),(0.76,0.42,0.33))$ \\
$\lambda_{3}$ & $((0.81,0.16,0.22),(0.86,0.11,0.13))$ & $((0.61,0.42,0.43),(0.76,0.32,0.22))$ \\
$\lambda_{4}$ & $((0.67,0.15,0.39),(0.91,0.11,0.14))$ & $((0.57,0.25,0.42),(0.75,0.20,0.33))$ \\
\hline
\end{tabular}

TABLE 22: Ranking results.

\begin{tabular}{lccccc}
\hline & \multicolumn{2}{c}{ Score values of alternatives } & $\lambda_{4}$ & Ranking \\
& $\lambda_{1}$ & $\lambda_{2}$ & $\lambda_{3}$ & 0.8432 & $\lambda_{3}<\lambda_{2}<\lambda_{1}<\lambda_{4}$ \\
\hline TOPSIS technique & 0.5959 & 0.4378 & 0.0796 & 0.62
\end{tabular}

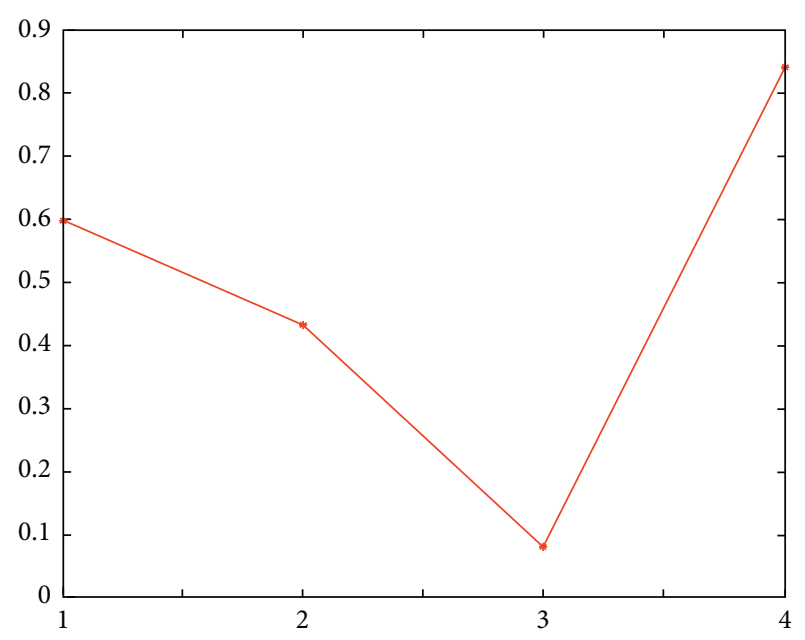

FIGURE 3: Graphical representation of alternatives SFR-TOPSIS method.

\section{Conclusion}

The application of the Internet in industry has broken down the conventional industry's heavily regulated industrial environment, allowing for intelligent manufacturing. Utilizing information technologies to improve conventional industrial organizations has been an essential trend in industrial enterprise development. Simultaneously, it implies that industrial firms would face new safety challenges. Since enterprise is more closely linked to the national economy and people's livelihoods, the authorities and the public focus primarily on ICS safety. In this paper, a new approach of spherical fuzzy rough aggregation operators has been developed to successfully resolve the issue of ICS security supplier selection. To demonstrate the applicability and rationality of the suggested approach, different test based on the aforementioned operators has been performed. According to the experimental findings, using the proposed technique to evaluate the ICS supplier is reasonable and acceptable. An addition, the comparison analysis performed by the developed models with the SFR-TOPSIS technique validates the capability, superiority, and reliability of the presented techniques.

In the future, we will aim to use the developed approach in more domains, as well as investigate advanced theories and algorithms in diverse fields.

\section{Data Availability}

The data used in the manuscript are hypothetical and can be used by anyone by just citing this article.

\section{Ethical Approval}

This article does not contain any studies with human participants or animals performed by any of the authors.

\section{Conflicts of Interest}

The authors declare that they have no conflicts of interest. 


\section{Authors' Contributions}

All authors have contributed equal to this article.

\section{References}

[1] M. Akram, A. Luqman, and J. C. R. Alcantud, "Risk evaluation in failure modes and effects analysis: hybrid TOPSIS and ELECTRE I solutions with Pythagorean fuzzy information," Neural Computing \& Applications, vol. 33, no. 11, pp. 56755703, 2021.

[2] S. Ashraf and S. Abdullah, "Spherical aggregation operators and their application in multiattribute group decision-making," International Journal of Intelligent Systems, vol. 34, no. 3, pp. 493-523, 2019.

[3] S. Ashraf, S. Abdullah, and S. Khan, "Fuzzy decision support modeling for internet finance soft power evaluation based on sine trigonometric Pythagorean fuzzy information," Journal of Ambient Intelligence and Humanized Computing, vol. 12, no. 12, pp. 3101-3119, 2020.

[4] S. Ashraf, T. Mahmood, S. Abdullah, and Q. Khan, "Different approaches to multi-criteria group decision making problems for picture fuzzy environment," Bulletin of the Brazilian Mathematical Society, New Series, vol. 50, no. 2, pp. 373-397, 2019.

[5] S. Ashraf, S. Abdullah, and M. Aslam, "Symmetric sum based aggregation operators for spherical fuzzy information: application in multi-attribute group decision making problem," Journal of Intelligent and Fuzzy Systems, vol. 38, no. 4, pp. 5241-5255, 2020.

[6] S. Ashraf, S. Abdullah, and A. O. Almagrabi, "A new emergency response of spherical intelligent fuzzy decision process to diagnose of COVID19," Soft Computing, pp. 1-17, 2020.

[7] S. Ashraf and S. Abdullah, "Emergency decision support modeling for COVID-19 based on spherical fuzzy information," International Journal of Intelligent Systems, vol. 35, no. 11, pp. 1601-1645, 2020.

[8] S. Ashraf, S. Abdullah, M. Aslam, M. Qiyas, and M. A. Kutbi, "Spherical fuzzy sets and its representation of spherical fuzzy t-norms and t-conorms," Journal of Intelligent and Fuzzy Systems, vol. 36, no. 6, pp. 6089-6102, 2019.

[9] L. F. Autran Monteiro Gomes and L. s. A. Duncan Rangel, "An application of the TODIM method to the multicriteria rental evaluation of residential properties," European Journal of Operational Research, vol. 193, no. 1, pp. 204-211, 2009.

[10] D. Pamučar and G. Ćirović, "The selection of transport and handling resources in logistics centers using Multi-Attributive Border Approximation Area Comparison (MABAC)," Expert Systems with Applications, vol. 42, no. 6, pp. 30163028, 2015.

[11] M. Keshavarz Ghorabaee, E. K. Zavadskas, L. Olfat, and Z. Turskis, "Multi-criteria inventory classification using a new method of evaluation based on distance from average solution (EDAS)," Informatica, vol. 26, no. 3, pp. 435-451, 2015.

[12] L.-1. Niu, J. Li, F. Li, and Z.-x. Wang, "Multi-criteria decisionmaking method with double risk parameters in intervalvalued intuitionistic fuzzy environments," Complex \& Intelligent Systems, vol. 6, no. 3, pp. 669-679, 2020.

[13] G. Wei, F. Lei, R. Lin et al., "Algorithms for probabilistic uncertain linguistic multiple attribute group decision making based on the GRA and CRITIC method: application to location planning of electric vehicle charging stations," Economic Research-Ekonomska Istraživanja, vol. 33, no. 1, pp. 828-846, 2020.
[14] M. Keshavarz Ghorabaee, E. K. Zavadskas, Z. Turskis, and J. Antucheviciene, "A new combinative distance-based assessment (CODAS) method for multi-criteria decisionmaking," Economic Computation \& Economic Cybernetics Studies \& Research, vol. 50, no. 3, 2016.

[15] S. Vluymans, D. Sánchez Tarragó, Y. Saeys, C. Cornelis, and F. Herrera, "Fuzzy rough classifiers for class imbalanced multi-instance data," Pattern Recognition, vol. 53, pp. 36-45, 2016.

[16] J. H. P. Paelinck, "Qualiflex: a flexible multiple-criteria method," Economics Letters, vol. 1, no. 3, pp. 193-197, 1978.

[17] T. He, G. Wei, J. Lu, J. Wu, C. Wei, and Y. Guo, "A novel EDAS based method for multiple attribute group decision making with Pythagorean 2-tuple linguistic information," Technological and Economic Development of Economy, vol. 26, no. 6, pp. 1125-1138, 2020.

[18] T. He, S. Zhang, G. Wei, R. Wang, J. Wu, and C. Wei, "CODAS method for 2-tuple linguistic Pythagorean fuzzy multiple attribute group decision making and its application to financial management performance assessment," Technological and Economic Development of Economy, vol. 26, no. 4, pp. 920-932, 2020.

[19] L. A. Zadeh, "Fuzzy sets," Information and Control, vol. 8, no. 3, pp. 338-353, 1965.

[20] K. T. Atanassov, "Intuitionistic fuzzy sets," Fuzzy Sets and Systems, vol. 20, no. 1, pp. 87-96, 1986.

[21] R. R. Yager, "Pythagorean fuzzy subsets," in Proceedings of the 2013 Joint IFSA World Congress and NAFIPS Annual Meeting (IFSA/NAFIPS), pp. 57-61, IEEE, Edmonton, Canada, June 2013.

[22] A. Çalık, "A novel Pythagorean fuzzy AHP and fuzzy TOPSIS methodology for green supplier selection in the Industry 4.0 era," Soft Computing, vol. 25, no. 3, pp. 2253-2265, 2021.

[23] A. Guleria and R. K. Bajaj, "A robust decision making approach for hydrogen power plant site selection utilizing $(\mathrm{R}, \mathrm{S})$ Norm Pythagorean Fuzzy information measures based on VIKOR and TOPSIS method," International Journal of Hydrogen Energy, vol. 45, no. 38, pp. 18802-18816, 2020.

[24] L. Li, R. Zhang, J. Wang, X. Zhu, and Y. Xing, "Pythagorean fuzzy power Muirhead mean operators with their application to multi-attribute decision making," Journal of Intelligent and Fuzzy Systems, vol. 35, no. 2, pp. 2035-2050, 2018.

[25] L. Wang and H. Garg, "Algorithm for multiple attribute decision-making with interactive archimedean norm operations under pythagorean fuzzy uncertainty," International Journal of Computational Intelligence Systems, vol. 14, no. 1, pp. 503-527, 2021.

[26] R. R. Yager, "Generalized orthopair fuzzy sets," IEEE Transactions on Fuzzy Systems, vol. 25, no. 5, pp. 1222-1230, 2016.

[27] B. C. Cuong and V. Kreinovich, "Picture Fuzzy Sets-a new concept for computational intelligence problems," in Proceedings of the 2013 third world congress on information and communication technologies (WICT 2013), pp. 1-6, IEEE, Hanoi, Vietnam, December 2013.

[28] S. V. Manemaran and R. Nagarajan, "Temporal generated N-picture fuzzy soft dimensions via algeberaic structures," International Journal of Research and Analytical Reviews, vol. 5, no. 4, pp. i660-i666, 2018.

[29] M. Khan, P. Kumam, S. Ashraf, and W. Kumam, "Generalized picture fuzzy soft sets and their application in decision support systems," Symmetry, vol. 11, no. 3, p. 415, 2019.

[30] S. Ashraf, S. Abdullah, T. Mahmood, and M. Aslam, "Cleaner production evaluation in gold mines using novel distance 
measure method with cubic picture fuzzy numbers," International Journal of Fuzzy Systems, vol. 21, no. 8, pp. 24482461, 2019.

[31] S. Abdullah and S. Ashraf, "Cubic picture fuzzy sets and their application to a petroleum circulation center evaluation problem," Sigma: Linguistique Anglaise, Linguistique Generale, vol. 10, no. 2, pp. 99-133, 2019.

[32] F. Kutlu Gündoğdu and C. Kahraman, "Spherical fuzzy sets and spherical fuzzy TOPSIS method," Journal of Intelligent \& Fuzzy Systems, vol. 36, no. 1, pp. 337-352, 2019.

[33] C. Kahraman, B. Oztaysi, S. C. Onar, and I. Otay, "q-spherical fuzzy sets and their usage in multi-attribute decision making," in Proceedings of the Developments of Artificial Intelligence Technologies in Computation and Robotics: Proceedings of the 14th International FLINS Conference (FLINS 2020), pp. 217225, Cologne, Germany, August 2020.

[34] S. Zeng, M. Munir, T. Mahmood, and M. Naeem, "Some T-spherical fuzzy Einstein interactive aggregation operators and their application to selection of photovoltaic cells," Mathematical Problems in Engineering, 2020.

[35] Z. a. Pawlak, "Rough sets," International Journal of Computer \& Information Sciences, vol. 11, no. 5, pp. 341-356, 1982.

[36] A. M. Radzikowska and E. E. Kerre, "A comparative study of fuzzy rough sets," Fuzzy Sets and Systems, vol. 126, no. 2, pp. 137-155, 2002.

[37] W. Pan, K. She, and P. Wei, "Multi-granulation fuzzy preference relation rough set for ordinal decision system," Fuzzy Sets and Systems, vol. 312, pp. 87-108, 2017.

[38] Y. Li, S. Wu, Y. Lin, and J. Liu, "Different classes' ratio fuzzy rough set based robust feature selection," Knowledge-Based Systems, vol. 120, pp. 74-86, 2017.

[39] T. Feng, H.-T. Fan, and J.-S. Mi, "Uncertainty and reduction of variable precision multigranulation fuzzy rough sets based on three-way decisions," International Journal of Approximate Reasoning, vol. 85, pp. 36-58, 2017.

[40] B. Sun, W. Ma, X. Chen, and X. Zhang, "Multigranulation vague rough set over two universes and its application to group decision making," Soft Computing, vol. 23, no. 18, pp. 8927-8956, 2019.

[41] C. Liu, W. Pedrycz, F. Jiang, and M. Wang, "Decision-theoretic rough set approaches to multi-covering approximation spaces based on fuzzy probability measure," Journal of Intelligent and Fuzzy Systems, vol. 34, no. 3, pp. 1917-1931, 2018.

[42] H. Zhang and L. Shu, "Generalized interval-valued fuzzy rough set and its application in decision making," International Journal of Fuzzy Systems, vol. 17, no. 2, pp. 279-291, 2015.

[43] H. Zhang, L. Shu, S. Liao, and C. Xiawu, "Dual hesitant fuzzy rough set and its application," Soft Computing, vol. 21, no. 12, pp. 3287-3305, 2017.

[44] C. Y. Wang and B. Q. Hu, "Granular variable precision fuzzy rough sets with general fuzzy relations," Fuzzy Sets and Systems, vol. 275, pp. 39-57, 2015.

[45] S. Vluymans, D. Sánchez Tarragó, Y. Saeys, C. Cornelis, and F. Herrera, "Fuzzy rough classifiers for class imbalanced multi-instance data," Pattern Recognition, vol. 53, pp. 36-45, 2016.

[46] T. Shaheen, M. I. Ali, and M. Shabir, "Generalized hesitant fuzzy rough sets (GHFRS) and their application in risk analysis," Soft Computing, pp. 1-13, 2020.

[47] M. A. Khan, S. Ashraf, S. Abdullah, and F. Ghani, "Applications of probabilistic hesitant fuzzy rough set in decision support system," Soft Computing, vol. 24, no. 22, pp. 16759-16774, 2020.
[48] G. Tang, F. Chiclana, and P. Liu, “A decision-theoretic rough set model with q-rung orthopair fuzzy information and its application in stock investment evaluation," Applied Soft Computing, vol. 91, Article ID 106212, 2020.

[49] D. Liang and W. Cao, "q-Rung orthopair fuzzy sets-based decision-theoretic rough sets for three-way decisions under group decision making," International Journal of Intelligent Systems, vol. 34, no. 12, pp. 3139-3167, 2019.

[50] Z. Zhang and S.-M. Chen, "Group decision making with incomplete q-rung orthopair fuzzy preference relations," Information Sciences, vol. 553, pp. 376-396, 2021.

[51] A. Hussain, M. Irfan Ali, and T. Mahmood, "Covering based q-rung orthopair fuzzy rough set model hybrid with TOPSIS for multi-attribute decision making," Journal of Intelligent and Fuzzy Systems, vol. 37, no. 1, pp. 981-993, 2019.

[52] X. Wang and E. Triantaphyllou, "Ranking irregularities when evaluating alternatives by using some ELECTRE methods," Omega, vol. 36, no. 1, pp. 45-63, 2008.

[53] C.-N. Huang, J. J. H. Liou, H.-W. Lo, and F.-J. Chang, "Building an assessment model for measuring airport resilience," Journal of Air Transport Management, vol. 95, Article ID 102101, 2021.

[54] H.-W. Lo, C.-C. Hsu, C.-N. Huang, and J. J. H. Liou, “An ITARA-TOPSIS based integrated assessment model to identify potential product and system risks," Mathematics, vol. 9, no. 3, p. 239, 2021.

[55] H.-W. Lo, J. J. H. Liou, C.-N. Huang, and Y.-C. Chuang, "A novel failure mode and effect analysis model for machine tool risk analysis," Reliability Engineering \& System Safety, vol. 183, pp. 173-183, 2019.

[56] M. Lin, C. Huang, Z. Xu, and R. Chen, "Evaluating IoT platforms using integrated probabilistic linguistic MCDM method," IEEE Internet of Things Journal, vol. 7, no. 11, pp. 11195-11208, 2020.

[57] V. C. Maduekwe and S. A. Oke, "Novel Taguchi schemebased DEMATEL methods and DEMATEL method for the principal performance indicators of maintenance in a food processing industry," International Journal of Intelligent Computing and Cybernetics, vol. 14, no. 3, pp. 363-397, 2021.

[58] X. Xiuqin, X. Jialiang, Y. Na, and W. Honghui, "Probabilistic uncertain linguistic TODIM method based on the generalized Choquet integral and its application," International Journal of Intelligent Computing and Cybernetics, vol. 14, no. 2, pp. 122-144, 2021. 